\title{
The physiological effects of hypobaric hypoxia versus normobaric hypoxia: a systematic review of crossover trials
}

\author{
Jonny Coppel ${ }^{1 *}$, Philip Hennis ${ }^{1}$, Edward Gilbert-Kawai ${ }^{1}$ and Michael PW Grocott ${ }^{1,2,3,4}$
}

\begin{abstract}
Much hypoxia research has been carried out at high altitude in a hypobaric hypoxia $(\mathrm{HH})$ environment. Many research teams seek to replicate high-altitude conditions at lower altitudes in either hypobaric hypoxic conditions or normobaric hypoxic $(\mathrm{NH})$ laboratories. Implicit in this approach is the assumption that the only relevant condition that differs between these settings is the partial pressure of oxygen $\left(\mathrm{PO}_{2}\right)$, which is commonly presumed to be the principal physiological stimulus to adaptation at high altitude. This systematic review is the first to present an overview of the current available literature regarding crossover studies relating to the different effects of $\mathrm{HH}$ and $\mathrm{NH}$ on human physiology. After applying our inclusion and exclusion criteria, 13 studies were deemed eligible for inclusion. Several studies reported a number of variables (e.g. minute ventilation and NO levels) that were different between the two conditions, lending support to the notion that true physiological difference is indeed present. However, the presence of confounding factors such as time spent in hypoxia, temperature, and humidity, and the limited statistical power due to small sample sizes, limit the conclusions that can be drawn from these findings. Standardisation of the study methods and reporting may aid interpretation of future studies and thereby improve the quality of data in this area. This is important to improve the quality of data that is used for improving the understanding of hypoxia tolerance, both at altitude and in the clinical setting.
\end{abstract}

Keywords: Normobaric hypoxia, Hypobaric hypoxia, Altitude

\section{Background}

Hypoxia research has numerous applications. These include investigating the pathogenesis and developing treatments for medical conditions characterised by hypoxia [1] and acute high altitude illness [2], as well as setting optimum training regimes for athletes [3].

Much hypoxia research has been carried out at high altitude in a hypobaric hypoxia $(\mathrm{HH})$ environment. Such 'field' studies present practical and logistical challenges including safety concerns about carrying out invasive procedures in a remote setting. For these reasons, many research teams seek to replicate high-altitude conditions at lower altitudes in either hypobaric hypoxic conditions or normobaric hypoxic $(\mathrm{NH})$ laboratories. In these two

\footnotetext{
* Correspondence: jonny.coppel@gmail.com

'University College London Centre for Altitude Space and Extreme Environment Medicine, UCLH NIHR Biomedical Research Centre, Institute of Sport and Exercise Health, 170 Tottenham Court Road, London W1T 7HA, UK Full list of author information is available at the end of the article
}

conditions, the hypoxic dose is calculated by the combination of the various barometric pressures $x$ inspired fraction of oxygen [4]. As emphasised in Conkin's 'Critique of the equivalent air altitude model' [5], implicit in this approach is the assumption that the only relevant condition that differs between these settings is the partial pressure of oxygen $\left(\mathrm{PO}_{2}\right)$, which is commonly presumed to be the principal physiological stimulus to adaptation at high altitude [6]. Although this assumption underpins the interpretation of many studies that form the basis of hypoxia physiology, it remains open to question as recently highlighted by Millet et al. [7] and controversy exists relating to the sporadic data in this area with various opinions on the matter as discussed in a recent series of 'point-counterpoints' [8]. The notion that $\mathrm{HH}$ and $\mathrm{NH}$ environments are interchangeable in terms of their effect on physiological responses is not proven.

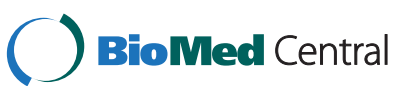

(C) 2015 Coppel et al.; licensee BioMed Central. This is an Open Access article distributed under the terms of the Creative Commons Attribution License (http://creativecommons.org/licenses/by/4.0), which permits unrestricted use, distribution, and reproduction in any medium, provided the original work is properly credited. The Creative Commons Public Domain Dedication waiver (http://creativecommons.org/publicdomain/zero/1.0/) applies to the data made available in this article unless otherwise stated. 
The practical outcomes of this debate affect a variety of fields. Many national teams in various sports incorporate altitude or hypoxic training into their programmes to aid haematological adaptations [3]. Additionally, armies across the world employ pre-acclimatisation strategies to train troops for deployment at high altitude [9]. This issue also applies to medical research such as therapeutic intermittent hypoxic methods [10] or critical care research into tissue hypoxia [1]. Thus, understanding the different impacts of $\mathrm{NH}$ and $\mathrm{HH}$ on physiology is important.

The aim of this systematic review is to conduct a comprehensive systematic literature search to address the questions: do humans react differently to $\mathrm{HH}$ when compared with $\mathrm{NH}$ (when evaluated in studies with a crossover design)?

\section{Review: methods}

\section{Criteria for considering studies for this review}

Candidate studies were identified using the following criteria (Figure 1).

\section{Types of studies}

We searched for primary research articles describing crossover trials comparing physiological responses to $\mathrm{NH}$ and $\mathrm{HH}$. Only crossover trials were considered due to the large inter-subject variation in their response to hypoxia.

\section{Types of participants}

We included studies involving lowland (defined as permanently living in locations $<2,000 \mathrm{~m}$ ) human subjects of any age who were not acclimatised to high altitude.

\section{Types of interventions}

We compared $\mathrm{NH}$ and $\mathrm{HH}$. $\mathrm{NH}$ and $\mathrm{HH}$ must be calculated to be equivalent to the same altitude. We included studies investigating any duration of exposure, and the
$\mathrm{HH}$ may have been performed either at sea level in a hypobaric chamber or at high altitude.

\section{Types of outcome measures}

Outcome variables were any human physiological response to atmospheric hypoxia. These responses included common phenotypes of interest in high altitude literature including (but not limited to) ventilation, hypoxaemia, exercise metabolism, nitric oxide (NO) production, osmotic balance, erythropoiesis and high-altitude illness.

\section{Search methods for identification of studies \\ Search strategy}

We attempted to identify all relevant trials regardless of language or publication status (published, unpublished, in press, and in progress). A literature search was carried out using the search engines Embase (all to date), Medline (performed on 15 October 2013) and Web of Science (performed on 15 October 2013). Snowballing was carried out; thus, the reference lists of all the shortlisted studies were checked for possible eligible studies.

\section{Search terms}

Search terms include ('Hypobari" hypoxia' OR 'simulated altitude' OR 'hypobari* anoxia') AND ('normbari* hypoxia' OR 'sea level hypoxia' OR 'sea-level hypoxia' OR 'normobari* anoxia').

\section{Data collection and analysis Selection of studies}

Titles and abstracts of candidate studies were screened for eligibility and duplicate references independently by two authors (JC and EG). The reasons for study exclusion were independently documented. For those papers that could not be excluded based on their titles and abstracts, the full paper was read to confirm eligibility. We resolved disagreements by consulting a third author

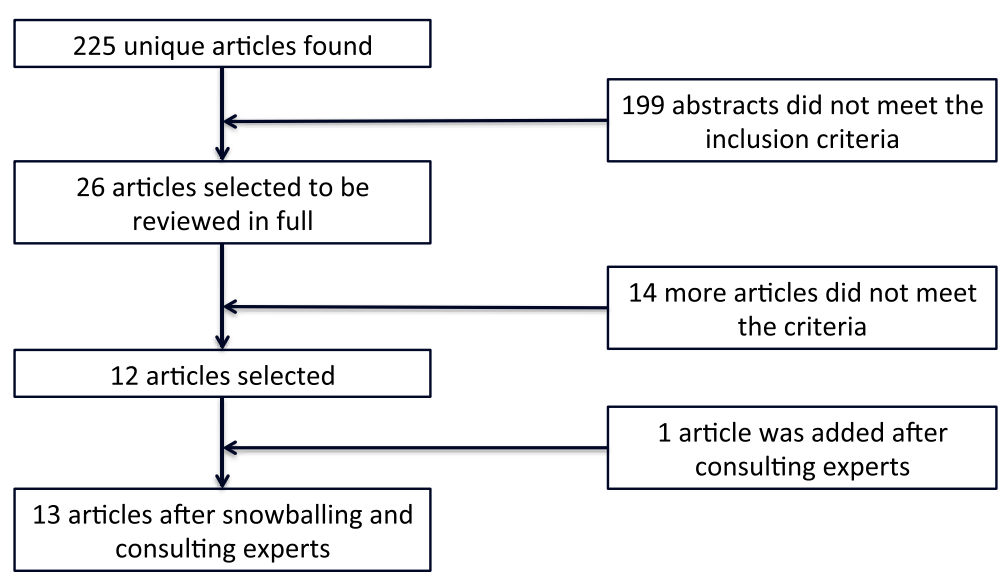

Figure 1 Methodology flow chart. This flow chart shows search methodology and results. 
$(\mathrm{PH})$ who arbitrated on inclusion. We obtained the assistance of translators when abstracts were not available in English.

\section{Data extraction and management}

Using data extraction forms, JC extracted information from each study and EG crosschecked the data. Data fields within the data extraction forms were directly linked to the formulated review question and planned assessment of included studies.

The data extraction forms contained the following information: study reference and reviewer identity, verification of study eligibility, study characteristics, study quality (see Quality of data section below), research methods, participants, intervention, outcome measures, results, and additional information.

\section{Assessment of risk of bias and study quality in included studies}

The risk of bias was independently assessed by JC and EG. Studies that do not report statistical significance ( $P$ values) for reported results were included, but their results were considered either as high risk of bias or unclear. In terms of study quality, we assessed the following: randomisation of subjects for the order of the crossover and whether they were blinded, length of washout period, presence/absence of sample size calculations, whether the statistical analyses accounted for the increased risk of type I errors when analysing large numbers of variables (adjustment for multiple comparisons), test-retest reliability, normalisation of testing environments for humidity $\left(\mathrm{pH}_{2} \mathrm{O}\right)$ as this can impact hypoxic dose calculations [11], and control of carbon dioxide $\left(\mathrm{CO}_{2}\right)$ in the testing environments.

\section{Data synthesis}

The results were tabulated and compared. No statistical analysis was carried out because the heterogeneity of the studies was such that the data could not be pooled (see below). Studies were categorised according to duration of hypoxic exposure. One hour was used as the cut off between 'long' and 'short' studies. All variables identified through our search strategy are highlighted in Table 1 (List of variables) but due to the number of variables reported, not all are considered in the written results and discussion. Emphasis is placed on the major physiological variables and those that are reported in more than one study. This was because a consistent result across multiple independent studies suggests validity of the finding. Additionally, when study characteristics were being determined, if a study did not mention a design feature, such as randomization of exposure order, it was assumed that the feature was not present. The results for each variable and time point can be found in Tables 2, 3, 4, 5, 6.

\section{Review: results Studies}

A total of 225 unique articles were identified in the EMBASE, MEDLINE and Web of Science searches. After applying our inclusion and exclusion criteria, 13 studies were deemed eligible for inclusion (Table 2: Study design). A total of 153 subjects were included in our review. Of these, six subjects were women and nine had chronic airflow limitations. One study was added after snowballing [12].

The studies investigated simulated altitudes from $1,700 \mathrm{~m}$ to $7,620 \mathrm{~m}$, and exposure to the hypoxia lasted between $5 \mathrm{~min}$ to $24 \mathrm{~h}$. The 13 studies were carried out in seven different countries: Australia (1), Japan (1), Spain (1), Sweden (1), Switzerland (2), France (2), and America (5).

\section{Variables measured}

\section{Quality of data}

Study design Nine studies [12-20] randomised the order of the crossover. Three studies $[6,21,22]$ did not, and one [23] was ambiguous as to whether randomization was used or not. Three studies $[12,18,22]$ had a washout period of at least 14 days, six studies [13-17,21] used 7 days, and four studies $[6,19,20,23]$ used less than $24 \mathrm{~h}$.

The largest study involved 20 people, the smallest 7 , and the mean was 12 . None of the studies stated they had conducted a sample size calculation to justify their chosen number. Only two studies $[12,23]$ mentioned accounting for the inflated risk of type I errors that arises when multiple comparisons are made, and both of these performed Bonferroni adjustments. Only one study measured the test-retest reliability of their outcome variable [23]. They performed an intra-class coefficient correlation and showed a good reliability of the postural stability measurements.

The methods used to control the degree of hypoxia administered in each study varied. Five studies mentioned controlling the relative humidity between $\mathrm{HH}$ and $\mathrm{NH}$. Of these, three $[15,17,18]$ maintained $50 \% \mathrm{hu}-$ midity $( \pm 1 \%)$ and two $[13,14]$ maintained it between $45 \%$ and $55 \%$. One paper [6] specifically mentioned the measurement and control of $\mathrm{CO}_{2}$ levels in the chambers using $\mathrm{CO}_{2}$ scrubbers.

\section{Ventilation}

Eight studies were identified that reported ventilation and oxygenation. Five of these lasted $\geq 1 \mathrm{~h}$ (long studies) $[12,13,15,22,16]$, and three lasted $<1 \mathrm{~h}$ (short studies) $[17,18,21]$. Five out of seven studies reporting minute ventilation reported values that were significantly lower in $\mathrm{HH}[12,13,17,21,22]$ (by up to $4 \mathrm{~L} / \mathrm{min}$ ) [13] (Figure 2: Graph of minute ventilation), whereas two identified no difference between conditions $[15,18]$. Consistent with 
Table 1 List of variables

\begin{tabular}{|c|c|c|c|}
\hline Oxygenation and ventilation & Cardiovascular & $\begin{array}{l}\text { AMS (acute mountain sickness) } \\
\text { and neurology }\end{array}$ & Other \\
\hline Ventilation (VE) & Hypoxic cardiac response (HCR) & Postural stability & Sweat rates \\
\hline Tidal volume (VT) & & & Forced vascular conductance \\
\hline Respiratory rate (Bf) & Heart rate variability (LH/HF) & Lake Louise AMS scores & $\begin{array}{l}\text { Oesophageal temperature thresholds } \\
\text { for increasing forearm skin vascular } \\
\text { conductance }\end{array}$ \\
\hline \multirow[t]{2}{*}{$\begin{array}{l}\text { Exhaled nitric oxide (NO) levels } \\
\text { (exNO) }\end{array}$} & Heart rate $(\mathrm{HR})$ & & $\begin{array}{l}\text { Oesophageal temperature thresholds } \\
\text { for increased sweat rate }\end{array}$ \\
\hline & & & Oesophageal temperature \\
\hline \multirow{3}{*}{$\begin{array}{l}\text { End tidal partial pressure of oxygen } \\
\left(\mathrm{PetO}_{2}\right)\end{array}$} & Stroke volume (SV) & & Skin temperature \\
\hline & & & Urine volume \\
\hline & Cardiac output (CO) & & Plasma volume \\
\hline \multirow{5}{*}{$\begin{array}{l}\text { End tidal partial pressure of carbon } \\
\text { dioxide }\left(\mathrm{P}_{\mathrm{ET}} \mathrm{CO}_{2}\right)\end{array}$} & & & Glomerular filtration rate (GFR) \\
\hline & & & Plasma potassium concentration (plasma $\mathrm{K}^{+}$) \\
\hline & Blood pressure (BP) & & Plasma sodium concentration (plasma $\mathrm{Na}^{+}$) \\
\hline & & & Plasma renin activity \\
\hline & & & Plasma aldosterone \\
\hline Alveolar ventilation (VA) & & & Free water clearance \\
\hline \multirow[t]{4}{*}{ Volume of $\mathrm{CO}_{2}$ produced $\left(\mathrm{VCO}_{2}\right)$} & & & Adrenocorticotropic hormone (ACTH) \\
\hline & & & Anti-diuretic hormone (ADH) \\
\hline & & & Anti-naturetic protein (ANP) \\
\hline & & & Blood base excess \\
\hline \multirow[t]{3}{*}{ Volume of oxygen consumed $\left(\mathrm{VO}_{2}\right)$} & & & Urine sodium-potassium ratio (urine $\mathrm{Na}^{+} / \mathrm{K}^{+}$) \\
\hline & & & Catecholamines, plasma osmolarity \\
\hline & & & $\mathrm{PH}$ \\
\hline \multirow[t]{6}{*}{ End tidal fraction of oxygen $\left(\mathrm{FetO}_{2}\right)$} & & & Urine osmolarity \\
\hline & & & Plasma lactate levels \\
\hline & & & Blood NO metabolites \\
\hline & & & Glutathione peroxidase (GPX) \\
\hline & & & MDA \\
\hline & & & Nitrotyrosine \\
\hline End tidal fraction of oxygen $\left(\mathrm{FetCO}_{2}\right)$ & & & $\begin{array}{l}\text { Plasma advanced oxidation protein } \\
\text { products and superoxide dismutase }\end{array}$ \\
\hline Duration of inspiration and expiration & & & Haematocrit (Hct) \\
\hline Hypoxic ventilatory response (HVR) & & & Haemoglobin concentration ( $\mathrm{Hb}$ conc) \\
\hline \multicolumn{4}{|l|}{ Respiratory quotient (RQ) } \\
\hline \multicolumn{4}{|l|}{ Peripheral oxygen saturations $\left(\mathrm{SpO}_{2}\right)$} \\
\hline \multicolumn{4}{|l|}{ Arterial oxygen saturations $\left(\mathrm{SaO}_{2}\right)$} \\
\hline \multicolumn{4}{|l|}{$\begin{array}{l}\text { Arterial oxygen and carbon dioxide } \\
\text { partial pressure }\left(\mathrm{P}_{\mathrm{a}} \mathrm{O}_{2}\right)\left(\mathrm{PaCO}_{2}\right)\end{array}$} \\
\hline \multicolumn{4}{|l|}{ Arterial oxygen content } \\
\hline Alveolar-arterial $\mathrm{PO}_{2}$ difference & & & \\
\hline
\end{tabular}

All the variables measured in the 13 accepted studies are listed. These have been subdivided into physiological systems.

this, the tidal volume was lower in $\mathrm{HH}$ in five out of six studies where this was reported (Figure 3: Graph of tidal volume) $[12,17,18,21,22]$. The largest difference in tidal volume found in a study was $0.9 \mathrm{~L}$ [12]. Two of the seven studies reporting breathing frequency found it to be higher in $\mathrm{HH}[17,18]$, whilst two others reported lower 
Table 2 Study design

\begin{tabular}{|c|c|c|c|c|c|c|c|}
\hline \multirow[t]{2}{*}{ Author and year } & \multirow[t]{2}{*}{ Type of outcome variable } & \multirow[t]{2}{*}{ Population } & \multicolumn{2}{|c|}{$\begin{array}{c}\mathrm{PiO}_{2} \text { of exposure (calculated by } \\
\left.\mathrm{PiO}_{2}=(\mathrm{Pb}-47) \times \mathrm{FiO}_{2}[4]\right)(\mathrm{mmHg})\end{array}$} & \multirow{3}{*}{$\begin{array}{l}\text { Duration of exposure } \\
\text { (h:min) }\end{array}$} & \multirow{2}{*}{$\begin{array}{l}\text { Randomised } \\
(\mathrm{Y}, \mathrm{N})\end{array}$} & \multirow{2}{*}{$\begin{array}{l}\text { Washout period } \\
\text { between trials (days:h) }\end{array}$} \\
\hline & & & $\mathrm{NH} \mathrm{HH}$ & & & & \\
\hline Basualto-Alarcon 2012 & $\begin{array}{l}\text { Ventilatory and } \\
\text { cardiovascular + exercise }\end{array}$ & 7 men & $3,000 \mathrm{~m}^{c}$ & $3,000 \mathrm{~m}^{\mathrm{c}}$ & & $\mathrm{N}$ & 7 \\
\hline Degache 2012 & Postural stability & 12 men & 118 and 102 & 121 and 103 & 00:20-25 build ${ }^{\mathrm{a}}, 00: 30^{\mathrm{b}}$ & Nil stated & $<00: 24$ \\
\hline Hemmingsson 2009 & Exhaled NO & 6 men, 2 women & 103 and 81 & 99 and 75 & 00:10 at each altitude & Y & $<00: 12$ \\
\hline Loeppky 2005 & Fluid balance & 9 men & 81 & 80 & 00:05 build ${ }^{\mathrm{a}}$ 10:00 & Y & 7 \\
\hline Loeppky 1997* & Ventilatory and cardiovascular & 9 men & 81 & 80 & 00:05 build 10:00 $^{\mathrm{a}}$ & Y & 7 \\
\hline Miyagawa 2010 & $\begin{array}{l}\text { Ventilatory, cardiovascular } \\
\text { and sweat + exercise }\end{array}$ & 7 men & 93 & 97 & 00:30 build ${ }^{a}$ only for $\mathrm{HH}$ 01:40 & Y & $>6$ \\
\hline Naughton 1995 & Haematological + exercise & $\begin{array}{l}9 \text { subjects with chronic airflow } \\
\text { limitation (CAL) with } 6 \text { controls }\end{array}$ & 1,829 and $2,438 \mathrm{~m}^{c}$ & 117 and 108 & 00:12 build ${ }^{a}$ included 00:52:00 & Y & 00:02 \\
\hline Roach 1996 & AMS and cardiovascular & 9 men & $4,564 \mathrm{~m}^{\mathrm{c}}$ & 80 & 09:00 & Y & 7 \\
\hline Savourey 2003 & Ventilatory and cardiovascular & 18 men & $4,500 \mathrm{~m}^{\mathrm{c}}$ & $4,500 \mathrm{~m}^{\mathrm{c}}$ & 00:10 build ${ }^{\mathrm{a}}, 00: 30$ & $\mathrm{Y}$ & 7 \\
\hline Savourey 2007 & Ventilatory and cardiovascular & 17 men, 1 woman & $4,500 \mathrm{~m}^{\mathrm{c}}$ & $4,500 m^{c}$ & 00:10 build ${ }^{\mathrm{a}}, 00: 30$ & $\mathrm{Y}$ & 14 \\
\hline Self 2011 & AMS and cardiovascular & 17 men and 3 women & $7,620 \mathrm{~m}^{c}$ & $7,620 \mathrm{~m}^{c}$ & 00:05 & $\mathrm{N}$ & $<00: 24$ \\
\hline Tucker 1983 & Mixed & 11 men & 82 & 80 & 00:15 build ${ }^{a}$ included 02:20 & $\mathrm{N}$ & Several weeks \\
\hline Faiss 2013 & $\begin{array}{l}\text { Exhaled NO, ventilatory and } \\
\text { cardiovascular + exercise }\end{array}$ & 10 men & 99 & 101 & 24:00:00 & Y & 23 \\
\hline
\end{tabular}

This table describes all the features of the accepted studies.

ahen the subjects entered the chamber, the environmental conditions were that of normal sea level but then were gradually made more hypoxic over the specified amount of time until the target hypoxic dose

was reached.
${ }^{\mathrm{b}}$ The different altitudes were tested consecutively. So sometimes the exposure was $1 \mathrm{~h}$ at 3,000 or $1,700 \mathrm{~m}$.

${ }^{\mathrm{c}} \mathrm{When} \mathrm{PiO}_{2}$ could not be calculated due to lack of information, the equivalent attitude estimated by the authors was given.

* $P$ values were for three conditions; in recovery no $P$ values unless stated in the discussion. 
Table 3 Oxygenation and ventilation variables

\begin{tabular}{|c|c|c|c|c|c|c|c|}
\hline Outcome & Author and year & $\begin{array}{c}\text { Duration of } \\
\text { exposure } \\
\text { (h:min) }\end{array}$ & $\begin{array}{l}\text { Hypobaric hypoxia } \\
\text { result [mean (SD)] }\end{array}$ & $\begin{array}{c}\text { Normobaric } \\
\text { hypoxia result } \\
{[\text { mean (SD)] }}\end{array}$ & $\begin{array}{l}\text { Difference } \\
\text { (HH - NH) }\end{array}$ & $P$ value & $\begin{array}{c}\text { Direction of } \\
\text { difference } \mathrm{NH} \\
\text { compared to } \mathrm{HH}\end{array}$ \\
\hline \multicolumn{8}{|l|}{ VE (L/min) } \\
\hline & Loeppky 1997 & 00:00 & 12.9 & 13.6 & - & - & - \\
\hline & Savourey 2007 & 00:10 build, 00:05 & $10.49(2.59)$ & $10.14(1.51)$ & - & $>0.05$ & NS \\
\hline & $\begin{array}{c}\text { Basualto-Alarcon } \\
2012\end{array}$ & $\begin{array}{l}\text { 00:15 build, 00:05 } \\
\text { acclimatisation }\end{array}$ & $10.5(4.9)$ & $10.3(1.8)$ & 0.2 & - & - \\
\hline & $\begin{array}{c}\text { Basualto-Alarcon } \\
2012\end{array}$ & $\begin{array}{l}\text { 00:15 build, 00:10 } \\
\text { acclimatisation }\end{array}$ & $35.7(5.9)$ & $39.7(6.7)$ & -4 & $<0.05$ & $\mathrm{NH}>\mathrm{HH}$ \\
\hline & Savourey 2003 & 00:10 build, 00:30 & - & - & - & $<0.02$ & $\mathrm{NH}>\mathrm{HH}$ \\
\hline & Savourey 2007 & 00:10 build, 00:30 & $10.70(1.93)$ & $10.78(1.93)$ & - & $>0.05$ & NS \\
\hline & Faiss 2013 & 01:00 & $13.6(1.8)$ & $13.3(3.3)$ & 0.3 & $>0.05$ & NS \\
\hline & Miyagawa $2010^{a}$ & 01:05 & 69.2 & 65.6 & - & $>0.05$ & NS \\
\hline & Miyagawa 2010 & 01:10 & 70.5 & 65.7 & - & $>0.05$ & NS \\
\hline & Miyagawa 2010 & 01:20 & 73.9 & 70.9 & - & $>0.05$ & NS \\
\hline & Miyagawa 2010 & 01:40 & 75.3 & 77 & - & $>0.05$ & NS \\
\hline & Tucker $1983^{b}$ & $\begin{array}{l}02: 20 \text { including } \\
\text { 00:15 build }\end{array}$ & 2.07 & 4.82 & -2.75 & - & - \\
\hline & Loeppky 1997 & 03:00 & 10.3 & 14.3 & -4.0 & $<0.01$ & $\mathrm{NH}>\mathrm{HH}$ \\
\hline & Loeppky 1997 & 06:00 & 10.6 & 12.7 & -2.1 & $<0.05$ & $\mathrm{NH}>\mathrm{HH}$ \\
\hline & Faiss 2013 & 08:00 & $11.8(1.9)$ & $14.9(3.5)$ & -3.1 & $<0.1$ & $\mathrm{NH}>\mathrm{HH}$ \\
\hline & & - & $10.7(1.8)$ & $12.2(1.6)$ & -1.5 & $<0.05$ & $\mathrm{NH}>\mathrm{HH}$ \\
\hline & & - & $12.7(2.3)$ & $14.2(1.5)$ & -1.5 & $<0.1$ & $\mathrm{NH}>\mathrm{HH}$ \\
\hline & Loeppky 1997 & 09:00 & 10.2 & 12.2 & - & $<0.05$ & $\mathrm{NH}>\mathrm{HH}$ \\
\hline & Loeppky 1997 & Recovery 12:00:00 & 9.2 & 10.1 & - & $>0.05$ & NS \\
\hline \multicolumn{8}{|l|}{$\mathrm{VT}(\mathrm{L})$} \\
\hline & Savourey 2007 & 00:10 build', 00:05 & $0.72(0.25)$ & $0.88(0.22)$ & - & 0.03 & $\mathrm{NH}>\mathrm{HH}$ \\
\hline & $\begin{array}{c}\text { Basualto-Alarcon } \\
2012\end{array}$ & $\begin{array}{l}\text { 00:15 build }{ }^{c}, 00: 05 \\
\text { acclimatisation }\end{array}$ & $0.81(0.36)$ & $0.82(0.21)$ & -0.01 & - & - \\
\hline & $\begin{array}{c}\text { Basualto-Alarcon } \\
2012\end{array}$ & $\begin{array}{l}\text { 00:15 build }{ }^{c}, 00: 10 \\
\text { acclimatisation }\end{array}$ & $1.85(0.56)$ & $1.91(0.53)$ & -0.06 & - & $\mathrm{NH}>\mathrm{HH}$ \\
\hline & Savourey 2003 & 00:10 build', 00:30 & - & - & - & $<0.001$ & $\mathrm{NH}>\mathrm{HH}$ \\
\hline & Savourey 2007 & 00:10 build', 00:30 & $0.83(0.37)$ & $0.86(0.34)$ & - & $>0.05$ & NS \\
\hline & Faiss 2013 & 01:00 & $0.88(0.21)$ & $0.89(0.26)$ & -0.01 & $>0.05$ & NS \\
\hline & Tucker $1983^{b}$ & $\begin{array}{l}\text { 02:20 including } \\
00: 15 \text { build }^{c}\end{array}$ & 106 & 152 & -46 & - & $\mathrm{NH}>\mathrm{HH}$ \\
\hline & Faiss 2013 & 08:00 & $0.75(0.21)$ & $0.94(0.3)$ & -0.19 & $<0.05$ & $\mathrm{NH}>\mathrm{HH}$ \\
\hline & Faiss 2013 & $16: 00$ & $0.75(0.23)$ & $0.84(0.24)$ & -0.9 & $<0.1$ & $\mathrm{NH}>\mathrm{HH}$ \\
\hline & Loeppky 1997 & 10:00 & - & - & - & $>0.05$ & NS \\
\hline & Faiss 2013 & $24: 00: 00$ & $0.86(0.25)$ & $0.95(0.23)$ & -0.09 & $<0.05$ & $\mathrm{NH}>\mathrm{HH}$ \\
\hline \multicolumn{8}{|c|}{ Bf (cycles/min) } \\
\hline & Savourey 2007 & 00:10 build', 00:05 & $15.73(4.64)$ & $12.24(3.80)$ & - & 0.03 & $\mathrm{HH}>\mathrm{NH}$ \\
\hline & $\begin{array}{c}\text { Basualto-Alarcon } \\
2012\end{array}$ & $\begin{array}{l}\text { 00:15 build }{ }^{c}, 00: 05 \\
\text { acclimatisation }\end{array}$ & $13.3(4.0)$ & $13.4(4.7)$ & -0.1 & - & - \\
\hline & $\begin{array}{c}\text { Basualto-Alarcon } \\
2012\end{array}$ & $\begin{array}{l}\text { 00:15 build }{ }^{c}, 00: 10 \\
\text { acclimatisation }\end{array}$ & $20.4(5.4)$ & $22.3(7.4)$ & -1.9 & $<0.05$ & $\mathrm{NH}>\mathrm{HH}$ \\
\hline & Savourey 2003 & 00:10 build', 00:30 & - & - & - & $<0.001$ & $\mathrm{HH}>\mathrm{NH}$ \\
\hline & Savourey 2007 & 00:10 build ${ }^{c}, 00: 30$ & $14.77(4.17)$ & $13.76(4.47)$ & - & $>0.05$ & NS \\
\hline
\end{tabular}


Table 3 Oxygenation and ventilation variables (Continued)

\begin{tabular}{|c|c|c|c|c|c|c|c|}
\hline & Faiss 2013 & 01:00 & $16.8(3.4)$ & $15.9(4.2)$ & -0.9 & $>0.05$ & NS \\
\hline & Miyagawa 2010 & 01:05 & 31 & 31 & - & $>0.05$ & NS \\
\hline & Miyagawa 2010 & $01: 10$ & 32 & 34 & - & $>0.05$ & NS \\
\hline & Miyagawa 2010 & $01: 20$ & 34 & 36 & - & $>0.05$ & NS \\
\hline & Miyagawa 2010 & $01: 40$ & 38 & 42 & - & $>0.05$ & NS \\
\hline & Tucker $1983^{b}$ & $\begin{array}{l}\text { 02:20 including } \\
\text { 00:15 build }\end{array}$ & 0.9 & 3.8 & -2.9 & - & $\mathrm{NH}>\mathrm{HH}$ \\
\hline & Faiss 2013 & 08:00 & $16.8(2.7)$ & $17.1(4.4)$ & -0.3 & $>0.05$ & NS \\
\hline & Loeppky 1997 & $10: 00$ & - & - & - & $>0.05$ & NS \\
\hline & Faiss 2013 & $16: 00$ & 16.1 (3) & $15.8(3.7)$ & -0.3 & $>0.05$ & NS \\
\hline & Faiss 2013 & $24: 00: 00$ & $16.8(3.8)$ & $16.2(3.8)$ & 0.6 & $>0.05$ & NS \\
\hline \multicolumn{8}{|l|}{$\mathrm{PetO}_{2}(\mathrm{mmHg})$} \\
\hline & Savourey 2007 & 00:10 build ${ }^{c}, 00: 05$ & 72.5 (6.58) & 79.56 (11.94) & - & 0.08 & $\begin{array}{c}\text { Borderline } \mathrm{NH}> \\
\mathrm{HH}\end{array}$ \\
\hline & Savourey 2003 & 00:10 build ${ }^{c}, 00: 30$ & - & - & - & $>0.05$ & NS \\
\hline & Savourey 2007 & 00:10 build ${ }^{c}, 00: 30$ & $73.15(7.16)$ & 76.09 (11.61) & - & 0.08 & $\begin{array}{c}\text { Borderline } \mathrm{NH}> \\
\mathrm{HH}\end{array}$ \\
\hline & Faiss 2013 & 01:00 & $66.4(4.1)$ & $62.3(2.8)$ & - & 4.1 & NS \\
\hline & Tucker $1983^{b}$ & $\begin{array}{l}\text { 02:20 including } \\
00: 15 \text { build }^{c}\end{array}$ & -36.1 & -32.6 & -3.5 & - & - \\
\hline & Faiss 2013 & $08: 00$ & $61.9(6.0)$ & $61.6(2.2)$ & 0.3 & $>0.05$ & NS \\
\hline & Loeppky 1997 & $10: 00$ & - & - & - & $>0.05$ & NS \\
\hline & Faiss 2013 & $16: 00$ & $65.0(5.4)$ & $62.7(2.6)$ & 2.3 & $>0.05$ & NS \\
\hline & Faiss 2013 & 24:00:00 & $65.6(5.5)$ & $65.6(2.8)$ & 0 & $>0.05$ & NS \\
\hline \multicolumn{8}{|l|}{$\operatorname{PetCO}_{2}(\mathrm{mmHg})$} \\
\hline & Savourey 2007 & 00:10 build, 00:05 & 44.09 (6.38) & $48.87(5.53)$ & - & 0.05 & $\begin{array}{c}\text { Borderline } \mathrm{NH}> \\
\mathrm{HH}\end{array}$ \\
\hline & Savourey 2003 & 00:10 build, $00: 30$ & - & - & - & $>0.05$ & NS \\
\hline & Savourey 2007 & 00:10 build, $00: 30$ & $43.43(6.02)$ & $46.13(6.61)$ & - & $>0.05$ & NS \\
\hline & Faiss 2013 & 01:00 & $33.4(2.5)$ & $29.4(2.4)$ & 4 & $<0.1$ & $\mathrm{HH}>\mathrm{NH}$ \\
\hline & Tucker $1983^{c}$ & $\begin{array}{l}\text { 02:20 including } \\
00: 15 \text { build }\end{array}$ & -2.8 & -3.6 & 0.8 & $>0.05$ & NS \\
\hline & Faiss 2013 & 08:00 & $33.8(2.1)$ & $27.5(1.3)$ & 6.3 & $<0.01$ & $\mathrm{HH}>\mathrm{NH}$ \\
\hline & Loeppky 1997 & 10:00 & - & - & -1.6 & $<0.02$ & $\mathrm{NH}>\mathrm{HH}$ \\
\hline & Faiss 2013 & $16: 00$ & $33.1(1.3)$ & $27.9(0.9)$ & 5.2 & $<0.01$ & $\mathrm{HH}>\mathrm{NH}$ \\
\hline & Faiss 2013 & 24:00:00 & $30.8(1.4)$ & $26.5(1.5)$ & 4.3 & $<0.01$ & $\mathrm{HH}>\mathrm{NH}$ \\
\hline \multirow[t]{5}{*}{$\begin{array}{l}\text { VA (alveolar } \\
\text { ventilation L/min) }\end{array}$} & Loeppky 1997 & 00:00 & 9.4 & 10.1 & - & - & - \\
\hline & Loeppky 1997 & 03:00 & 7.2 & 10.5 & $-46 \%$ & $<0.05$ & $\mathrm{NH}>\mathrm{HH}$ \\
\hline & Loeppky 1997 & 06:00 & 7.6 & 9.1 & - & $<0.05$ & $\mathrm{NH}>\mathrm{HH}$ \\
\hline & Loeppky 1997 & 09:00 & 7.6 & 9.2 & - & $<0.05$ & $\mathrm{NH}>\mathrm{HH}$ \\
\hline & Loeppky 1997 & Recovery 12:00:00 & 6.7 & 7.2 & - & - & - \\
\hline \multicolumn{8}{|l|}{$\mathrm{VCO}_{2} \mathrm{ml} / \mathrm{min}$} \\
\hline & Loeppky 1997 & 00:00 & 295 & 333 & - & - & - \\
\hline & Miyagawa 2010 & 01:05 & 2188 & 2108 & - & $>0.05$ & NS \\
\hline & Miyagawa 2010 & $01: 10$ & 2121 & 2007 & - & $>0.05$ & NS \\
\hline & Miyagawa 2010 & 01:20 & 2078 & 2060 & - & $>0.05$ & NS \\
\hline
\end{tabular}


Table 3 Oxygenation and ventilation variables (Continued)

\begin{tabular}{|c|c|c|c|c|c|c|c|}
\hline & Miyagawa 2010 & 01:40 & 2021 & 2082 & - & $>0.05$ & NS \\
\hline & Loeppky 1997 & 03:00 & 216 & 330 & - & $<0.05$ & $\mathrm{NH}>\mathrm{HH}$ \\
\hline & Loeppky 1997 & 06:00 & 227 & 296 & - & $<0.05$ & $\mathrm{NH}>\mathrm{HH}$ \\
\hline & Loeppky 1997 & 09:00 & 235 & 302 & -67 & $<0.05$ & $\mathrm{NH}>\mathrm{HH}$ \\
\hline & Loeppky 1997 & Recovery 12:00 & 241 & 267 & - & - & - \\
\hline \multicolumn{8}{|l|}{$\mathrm{VO}_{2}$ consumed } \\
\hline & Loeppky 1997 & 00:00 & 329 & 340 & - & - & - \\
\hline & Miyagawa 2010 & 01:05 & 1709 & 1611 & - & $>0.05$ & NS \\
\hline & Miyagawa 2010 & 01:10 & 1783 & 1637 & - & $>0.05$ & NS \\
\hline & Miyagawa 2010 & 01:20 & 1826 & 1748 & - & $>0.05$ & NS \\
\hline & Miyagawa 2010 & 01:40 & 1836 & 1840 & - & $>0.05$ & NS \\
\hline & Loeppky 1997 & 03:00 & 250 & 361 & - & $<0.05$ & $\mathrm{NH}>\mathrm{HH}$ \\
\hline & Loeppky 1997 & 06:00 & 262 & 319 & - & $<0.05$ & $\mathrm{NH}>\mathrm{HH}$ \\
\hline & Loeppky 1997 & 09:00 & 278 & 326 & - & $<0.05$ & $\mathrm{NH}>\mathrm{HH}$ \\
\hline & Loeppky 1997 & $12: 00$ & 301 & 291 & - & - & - \\
\hline \multicolumn{8}{|l|}{$\mathrm{FetO}_{2}$} \\
\hline & Savourey 2007 & 00:10 build ${ }^{c}, 00: 05$ & - & - & - & $>0.05$ & NS \\
\hline & Savourey 2003 & 00:10 build ${ }^{c}, 00: 30$ & - & - & - & $<0.00001$ & $\mathrm{HH}>\mathrm{NH}$ \\
\hline & Savourey 2007 & 00:10 build ${ }^{c}, 00: 30$ & - & - & - & $>0.05$ & NS \\
\hline \multicolumn{8}{|l|}{$\mathrm{FetCO}_{2}$} \\
\hline & Savourey 2007 & 00:10 build, 00:05 & - & - & - & $>0.05$ & NS \\
\hline & Savourey 2003 & 00:10 build, 00:30 & - & - & - & $<0.00001$ & $\mathrm{HH}>\mathrm{NH}$ \\
\hline & Savourey 2007 & 00:10 build, 00:30 & - & - & - & $>0.05$ & NS \\
\hline \multirow[t]{2}{*}{$\begin{array}{l}\text { Duration of } \\
\text { inspiration/s }\end{array}$} & Savourey 2007 & 00:10 build ${ }^{c}, 00: 05$ & $1.94(0.65)$ & $2.99(0.98)$ & - & 0.01 & $\mathrm{NH}>\mathrm{HH}$ \\
\hline & Savourey 2007 & 00:10 build', $00: 30$ & $2.40(1.25)$ & $3.00(1.16)$ & - & $>0.05$ & NS \\
\hline \multicolumn{8}{|l|}{ Duration of expiration/s } \\
\hline & Savourey 2007 & 00:10 build ${ }^{c}, 00: 05$ & $2.09(0.87)$ & $1.98(0.84)$ & - & $>0.05$ & NS \\
\hline & Savourey 2007 & 00:10 build ${ }^{c}, 00: 30$ & - & - & - & $>0.05$ & NS \\
\hline \multirow[t]{2}{*}{$\begin{array}{l}\text { Hypoxic ventilatory } \\
\text { response (HVR) } 1 \%^{-1}\end{array}$} & Savourey 2007 & 00:10 build ${ }^{c}, 00: 05$ & -0.05 & 0.03 & - & $>0.05$ & NS \\
\hline & Savourey 2007 & 00:10 build', 00:30 & -0.09 & -0.07 & - & $>0.05$ & NS \\
\hline \multicolumn{8}{|l|}{$\mathrm{SpO}_{2}(\%)$} \\
\hline & Savourey 2007 & 00:10 build', 00:05 & $83.03(4.49)$ & $87.11(4.81)$ & -4.08 & $<0.05$ & $\mathrm{NH}>\mathrm{HH}$ \\
\hline & $\begin{array}{c}\text { Basualto-Alarcon } \\
2012\end{array}$ & $\begin{array}{l}\text { 00:15 build }{ }^{c}, 00: 05 \\
\text { acclimatisation }\end{array}$ & $91.6(4.2)$ & $89.1(3.8)$ & 2.5 & $<0.05$ & $\mathrm{HH}>\mathrm{NH}$ \\
\hline & $\begin{array}{c}\text { Basualto-Alarcon } \\
2012\end{array}$ & $\begin{array}{l}\text { 00:15 build }{ }^{c}, 00: 10 \\
\text { acclimatisation }\end{array}$ & $85.3(3.8)$ & $86.0(1.7)$ & -0.7 & - & - \\
\hline & Savourey 2003 & 00:10 build', 00:30 & - & - & - & $<0.05$ & $\mathrm{NH}>\mathrm{HH}$ \\
\hline & Savourey 2007 & 00:10 build ${ }^{c}, 00: 30$ & $82.49(4.39)$ & $85.50(4.84)$ & -2.99 & 0.04 & $\mathrm{NH}>\mathrm{HH}$ \\
\hline & Faiss 2013 & 01:00 & $93(1)$ & $90(3)$ & 3 & $>0.05$ & NS \\
\hline & Tucker $1983^{b}$ & $\begin{array}{l}\text { 02:20 including } \\
00: 15 \text { build }^{c}\end{array}$ & -13.2 & -13.5 & 0.3 & $>0.05$ & NS \\
\hline & Faiss 2013 & 08:00 & $91(3)$ & $91(3)$ & 0 & $>0.05$ & NS \\
\hline & Roach 1996 & 09:00 & $83 \%(1 \%)$ & $83 \%(0.7 \%)$ & 0 & $>0.05$ & NS \\
\hline & Loeppky 2005 & 10:00 & $82 \%$ & $83 \%$ & $-1 \%$ & $>0.05$ & NS \\
\hline
\end{tabular}


Table 3 Oxygenation and ventilation variables (Continued)

\begin{tabular}{|c|c|c|c|c|c|c|c|}
\hline & Loeppky 1997 & $10: 00$ & - & - & - & $>0.05$ & NS \\
\hline & Faiss 2013 & $16: 00$ & $92(2)$ & $91(2)$ & 1 & $>0.05$ & NS \\
\hline & Faiss 2013 & 24:00:00 & $93(2)$ & $92(1)$ & 1 & $>0.05$ & NS \\
\hline \multicolumn{8}{|l|}{$\mathrm{SaO}_{2}(\%)$} \\
\hline & Self 2011 & 00:05 & - & - & - & 0.005 & $\mathrm{NH}>\mathrm{HH}$ \\
\hline & Savourey 2007 & 00:10 build ${ }^{c}, 00: 05$ & - & - & - & $>0.05$ & NS \\
\hline & Savourey 2003 & 00:10 build ${ }^{c}, 00: 30$ & $85 \%(4)$ & $88 \%(3)$ & -3 & $<0.05$ & $\mathrm{NH}>\mathrm{HH}$ \\
\hline & Savourey 2007 & 00:10 build ${ }^{c}, 00: 30$ & $81.09 \%(7.76)$ & $85.48 \%(5.63)$ & -4.39 & 0.07 & $\begin{array}{c}\text { Borderline } \mathrm{NH}> \\
\mathrm{HH}\end{array}$ \\
\hline & Miyagawa 2010 & 01:05 & 82 & 83 & -1 & $>0.05$ & NS \\
\hline & Miyagawa 2010 & 01:10 & 81 & 82 & -1 & $>0.05$ & NS \\
\hline & Miyagawa 2010 & 01:20 & 81 & 82 & -1 & $>0.05$ & NS \\
\hline & Miyagawa 2010 & 01:40 & 82 & 81 & 1 & $>0.05$ & NS \\
\hline & Roach 1996 & 09:00 & $83 \%(1 \%)$ & $83 \%(0.7 \%)$ & 0 & $>0.05$ & NS \\
\hline \multicolumn{8}{|l|}{$\mathrm{PaO}_{2}$} \\
\hline & Self 2011 & 00:05 & - & - & - & 0.004 & $\mathrm{HH}>\mathrm{NH}$ \\
\hline & Savourey 2007 & 00:10 build ${ }^{c}, 00: 05$ & - & - & - & $>0.05$ & NS \\
\hline & Savourey 2003 & 00:10 build ${ }^{c}, 00: 30$ & $6.38(0.60)$ & $6.90(0.86)$ & -0.52 & $\leq 0.05$ & $\begin{array}{c}\text { Borderline } \mathrm{NH}> \\
\mathrm{HH}\end{array}$ \\
\hline & Savourey 2007 & 00:10 build ${ }^{c}, 00: 30$ & - & - & - & $>0.05$ & NS \\
\hline CAL subjects & Naughton 1995 & $00: 52$ & - & - & -1.1 & $>0.05$ & NS \\
\hline Control subjects & Naughton 1995 & 00:52 & - & - & 0.7 & $>0.05$ & NS \\
\hline \multicolumn{8}{|l|}{$\mathrm{PaCO}_{2}$} \\
\hline & Self 2011 & 00:05 & - & - & - & 0.005 & $\mathrm{NH}>\mathrm{HH}$ \\
\hline & Savourey 2007 & 00:10 build, 00:05 & - & - & - & $>0.05$ & NS \\
\hline & Savourey 2003 & 00:10 build, 00:30 & $4.65(0.54)$ & $5.06(0.46)$ & -0.41 & $\leq 0.05$ & $\begin{array}{c}\text { Borderline } \mathrm{NH}> \\
\mathrm{HH}\end{array}$ \\
\hline & Savourey 2007 & 00:10 build, 00:30 & $46.3(6.5)$ & $52.2(4.2)$ & -5.9 & 0.005 & $\mathrm{NH}>\mathrm{HH}$ \\
\hline CAL subjects & Naughton 1995 & $00: 52$ & - & - & 0.3 & $>0.05$ & NS \\
\hline \multirow[t]{2}{*}{ Control subjects } & Naughton 1995 & 00:52 & - & - & -0.8 & $>0.05$ & NS \\
\hline & Tucker $1983^{b}$ & $\begin{array}{l}\text { 02:20 including } \\
\text { 00:15 build }\end{array}$ & -3.7 & -5.6 & 1.9 & - & - \\
\hline \multicolumn{8}{|c|}{$\begin{array}{l}\text { Alveolar-arterial } \mathrm{PO}_{2} \\
\text { difference }\end{array}$} \\
\hline CAL subjects & Naughton 1995 & $00: 52$ & - & - & 0.7 & $>0.05$ & NS \\
\hline Control subjects & Naughton 1995 & 00:52 & - & - & 0.2 & $>0.05$ & NS \\
\hline \multicolumn{8}{|c|}{ Arterial $\mathrm{O}_{2}$ content } \\
\hline & Savourey 2007 & 00:10 build ${ }^{c}, 00: 05$ & - & - & - & $>0.05$ & NS \\
\hline & Savourey 2007 & 00:10 build', 00:30 & - & - & - & $>0.05$ & NS \\
\hline
\end{tabular}

This table lists all the values of the measured variables that relate to oxygenation and ventilation.

NS insignificant, hyphen no values given, CAL Chronic Airflow Limitation.

${ }^{a}$ Exercise started at 01:00.

${ }^{b}$ Difference from controls.

'When the subjects entered the chamber, the environmental conditions were that of normal sea level but then were gradually made more hypoxic over the specified amount of time until the target hypoxic dose was reached.

values in $\mathrm{HH}[21,22]$ and there was no difference in the remainder [12,13,15] (Figure 4: Graph of breathing frequency). The only study that reported alveolar ventilation found that it was higher in $\mathrm{NH}$ than $\mathrm{HH}$ [13].

\section{Oxygenation}

The peripheral oxygen saturations measured by pulse oximetry $\left(\mathrm{SpO}_{2}\right)$ were significantly lower in $\mathrm{HH}$ in two out of three short studies $[17,18]$. One study found that 
Table 4 Cardiovascular variables

\begin{tabular}{|c|c|c|c|c|c|c|c|}
\hline Outcomes & Author and year & $\begin{array}{c}\text { Duration of } \\
\text { exposure (h:min) }\end{array}$ & $\begin{array}{l}\text { Hypobaric hypoxia } \\
\text { result [mean (SD)] }\end{array}$ & $\begin{array}{l}\text { Normobaric hypoxia } \\
\text { result [mean (SD)] }\end{array}$ & $\begin{array}{l}\text { Difference } \\
\text { (HH - NH) }\end{array}$ & $P$ value & $\begin{array}{c}\text { Direction of } \\
\text { difference NH } \\
\text { compared to } \mathrm{HH}\end{array}$ \\
\hline \multicolumn{8}{|c|}{$\begin{array}{l}\text { Hypoxic cardiac } \\
\text { response (HCR) } \\
\text { bpm \%-1 }\end{array}$} \\
\hline & Savourey 2007 & 00:10 build ${ }^{\mathrm{a}}, 00: 05$ & -0.61 & -0.63 & 0.02 & $>0.05$ & NS \\
\hline & Savourey 2007 & 00:10 build ${ }^{\mathrm{a}}, 00: 30$ & -0.52 & -0.79 & 0.27 & $>0.05$ & NS \\
\hline \multicolumn{8}{|l|}{ LF/HF\% } \\
\hline & Basualto-Alarcon 2012 & $\begin{array}{l}\text { 00:15 build }{ }^{\mathrm{a}}, 00: 10 \\
\text { acclimatisation }\end{array}$ & $1.96(2.6)$ & $1.28(0.92)$ & 0.68 & $<0.05$ & $\mathrm{HH}>\mathrm{NH}$ \\
\hline \multicolumn{8}{|l|}{$H R$} \\
\hline & Self 2011 & 00:01 & $104.9(14.3)$ & $96.6(14.6)$ & 8.3 & $<0.05$ & $\mathrm{HH}>\mathrm{NH}$ \\
\hline & Self 2011 & 00:04 & - & - & - & $>0.05$ & NS \\
\hline & Basualto-Alarcon 2012 & $\begin{array}{l}\text { 00:15 build }{ }^{\mathrm{a}}, 00: 05 \\
\text { acclimatisation }\end{array}$ & $61(9)$ & $62(6)$ & -1 & - & - \\
\hline & Basualto-Alarcon 2012 & $\begin{array}{l}\text { 00:15 build }{ }^{\mathrm{a}}, 00: 10 \\
\text { acclimatisation }\end{array}$ & $129(23)$ & $134(16)$ & -5 & $<0.05$ & $\mathrm{NH}>\mathrm{HH}$ \\
\hline & Savourey 2007 & 00:10 build ${ }^{\mathrm{a}}, 00: 05$ & $70.32(9.91)$ & $69.62(9.95)$ & & $>0.05$ & NS \\
\hline & Savourey 2003 & 00:10 build ${ }^{\mathrm{a}}, 00: 30$ & - & - & - & $<0.05$ & $\mathrm{HH}>\mathrm{NH}$ \\
\hline & Savourey 2007 & 00:10 build ${ }^{\mathrm{a}}, 00: 30$ & $69.50(12.07)$ & 70.67 (12.07) & - & $>0.05$ & NS \\
\hline & Faiss 2013 & 01:00 & $62(8)$ & $63(10)$ & -1 & $>0.05$ & NS \\
\hline & Miyagawa 2010 & 01:05 & 144 & 137 & - & $>0.05$ & NS \\
\hline & Miyagawa 2010 & 01:10 & 150 & 146 & - & $>0.05$ & NS \\
\hline & Miyagawa 2010 & 01:40 & 166 & 164 & - & $>0.05$ & NS \\
\hline & Tucker $1983^{\mathrm{a}}$ & $\begin{array}{l}\text { 02:20 including } \\
\text { 00:15 build }\end{array}$ & 10.4 & 3.6 & 6.8 & - & $\mathrm{HH}>\mathrm{NH}$ \\
\hline & Faiss 2013 & 08:00 & $68(13)$ & $69(13)$ & -1 & $>0.05$ & NS \\
\hline & Faiss 2013 & $16: 00$ & $61(10)$ & $66(7)$ & -5 & $>0.05$ & NS \\
\hline & Faiss 2013 & $24: 00$ & $65(9)$ & $71(10)$ & -6 & $>0.05$ & NS \\
\hline \multicolumn{8}{|c|}{ Stroke volume } \\
\hline & Miyagawa 2010 & 01:05 & 105 & 107 & -2 & $>0.05$ & NS \\
\hline & Miyagawa 2010 & 01:10 & 113 & 126 & -13 & $>0.05$ & NS \\
\hline & Miyagawa 2010 & $01: 40$ & 116 & 124 & -8 & $>0.05$ & NS \\
\hline \multicolumn{8}{|c|}{ Cardiac output } \\
\hline & Miyagawa 2010 & 01:05 & 15.3 & 14.7 & & $>0.05$ & NS \\
\hline & Miyagawa 2010 & 01:10 & 17.1 & 18.2 & & $>0.05$ & NS \\
\hline & Miyagawa 2010 & $01: 40$ & 19.4 & 20.1 & & $>0.05$ & NS \\
\hline \multicolumn{8}{|l|}{ Mean BP } \\
\hline & Miyagawa 2010 & 01:05 & 112 & 107 & & $>0.05$ & NS \\
\hline & Miyagawa 2010 & 01:10 & 108 & 107 & & $>0.05$ & NS \\
\hline & Miyagawa 2010 & 01:40 & 100 & 99 & & $>0.05$ & NS \\
\hline
\end{tabular}


Table 4 Cardiovascular variables (Continued)

\begin{tabular}{|c|c|c|c|c|c|c|c|}
\hline \\
\hline \multicolumn{8}{|c|}{$\begin{array}{l}\text { BP systolic (Iorr) } \\
\text { Faiss } 2013\end{array}$} \\
\hline \multicolumn{8}{|c|}{ acker 1983} \\
\hline & Faiss 2013 & 08:00 & $124(9)$ & $123(7)$ & 1 & $>0.05$ & NS \\
\hline & Faiss 2013 & 16:00 & $121(9)$ & $118(9)$ & 3 & $>0.05$ & NS \\
\hline & Faiss 2013 & 24:00:00 & $131(10)$ & $129(9)$ & 2 & $>0.05$ & NS \\
\hline
\end{tabular}

This table lists all the values of the measured variables that relate to the cardiovascular system.

NS insignificant.

${ }^{a}$ When the subjects entered the chamber, the environmental conditions were that of normal sea level but then were gradually made more hypoxic over the specified amount of time until the target hypoxic dose was reached.

${ }^{\mathrm{b}}$ Difference from controls.

Table 5 AMS and neurology variables

\begin{tabular}{|c|c|c|c|c|c|c|c|}
\hline Outcome & $\begin{array}{c}\text { Author and } \\
\text { year }\end{array}$ & $\begin{array}{c}\text { Duration of } \\
\text { exposure (h:min) }\end{array}$ & $\begin{array}{l}\text { Hypobaric hypoxia } \\
\text { result [mean (SD)] }\end{array}$ & $\begin{array}{l}\text { Normobaric hypoxia } \\
\text { result [mean (SD)] }\end{array}$ & $\begin{array}{l}\text { Difference } \\
\text { (HH - NH) }\end{array}$ & $P$ value & $\begin{array}{l}\text { Direction of difference } \\
\mathrm{NH} \text { compared to HH }\end{array}$ \\
\hline \multicolumn{8}{|l|}{$\begin{array}{l}\text { Length of centre } \\
\text { of pressure trajectory } \\
\text { in } Y \text { axis }\end{array}$} \\
\hline Eyes open 1,700 m & Degache 2012 & $00: 30$ & $114.2(38.8)$ & $129.5(53.3)$ & -15.3 & - & $\mathrm{NH}>\mathrm{HH}$ \\
\hline Eyes closed 1,700 m & Degache 2012 & $00: 30$ & $127.2(54.9)$ & $87.7(44.8)$ & 39.5 & - & $\mathrm{HH}>\mathrm{NH}$ \\
\hline Dual task $1,700 \mathrm{~m}$ & Degache 2012 & $00: 30$ & $128.7(87.1)$ & $79.9(30.3)$ & 48.8 & - & $\mathrm{HH}>\mathrm{NH}$ \\
\hline $\begin{array}{l}\text { Romberg's index } \\
1,700 \mathrm{~m}\end{array}$ & Degache 2012 & $00: 30$ & $1.35(0.19)$ & $1.42(0.34)$ & -0.07 & - & - \\
\hline Eyes open 3,000 m & Degache 2012 & $00: 30$ & $123.1(22.6)$ & $127.2(41.5)$ & -4.1 & - & - \\
\hline Eyes closed 3,000 m & Degache 2012 & $00: 30$ & $104.7(27.0)$ & $89.1(39.9)$ & 15.6 & - & $\mathrm{HH}>\mathrm{NH}$ \\
\hline Dual task 3,000 m & Degache 2012 & $00: 30$ & $91.9(22.4)$ & $82.4(30.4)$ & 9.5 & - & $\mathrm{HH}>\mathrm{NH}$ \\
\hline $\begin{array}{l}\text { Romberg's index } \\
3,000 \mathrm{~m}\end{array}$ & Degache 2012 & $00: 30$ & $1.33(0.22)$ & $1.39(0.29)$ & -0.06 & - & - \\
\hline \multicolumn{8}{|l|}{$\begin{array}{l}\text { Variance of speed } \\
\text { of CoP }\end{array}$} \\
\hline Eyes open 1,700 m & Degache 2012 & $00: 30$ & $111.0(56.2)$ & $151.4(30.2)$ & -40.4 & - & $\mathrm{NH}>\mathrm{NH}$ \\
\hline Eyes closed 1,700 m & Degache 2012 & $00: 30$ & $111.0(58.8)$ & $149.9(31.5)$ & -38.9 & - & $\mathrm{NH}>\mathrm{HH}$ \\
\hline Dual task $1,700 \mathrm{~m}$ & Degache 2012 & $00: 30$ & $112.1(57.7)$ & $151.1(31.7)$ & -39 & - & $\mathrm{NH}>\mathrm{HH}$ \\
\hline $\begin{array}{l}\text { Romberg's index } \\
1,700 \mathrm{~m}\end{array}$ & Degache 2012 & $00: 30$ & $0.98(0.09)$ & $0.99(0.03)$ & -0.01 & - & - \\
\hline Eyes open 3,000 m & Degache 2012 & $00: 30$ & $150.5(42.3)$ & $160.8(14.0)$ & -10.3 & - & $\mathrm{NH}>\mathrm{HH}$ \\
\hline Eyes closed 3,000 m & Degache 2012 & $00: 30$ & $142.9(40.8)$ & $158.6(13.7)$ & -15.7 & - & $\mathrm{NH}>\mathrm{HH}$ \\
\hline Dual task 3,000 m & Degache 2012 & $00: 30$ & $143.4(39.2)$ & $160.1(15.0)$ & -16.7 & - & $\mathrm{NH}>\mathrm{HH}$ \\
\hline $\begin{array}{l}\text { Romberg's index } \\
3,000 \mathrm{~m}\end{array}$ & Degache 2012 & $00: 30$ & $0.95(0.11)$ & $0.99(0.02)$ & -0.04 & - & - \\
\hline \multirow{4}{*}{$\begin{array}{l}\text { Lake Louise AMS } \\
\text { scores }\end{array}$} & Self 2011 & 00:01 & - & - & 2.36 & $>0.05$ & NS \\
\hline & Self 2011 & 00:04 & - & - & -4.89 & $>0.05$ & NS \\
\hline & Roach 1996 & 09:00 & - & - & - & $<0.01$ & $\mathrm{HH}>\mathrm{NH}$ \\
\hline & Loeppky 2005* & $10: 00$ & - & - & - & $<0.001$ & $\mathrm{HH}>\mathrm{NH}$ \\
\hline
\end{tabular}

This table lists all the values of the measured variables that relate to AMS and neurology.

NS insignificant.

*P value calculated including hypobaric normoxia. 
Table 6 Additional physiological variables

\begin{tabular}{|c|c|c|c|c|c|c|c|}
\hline Outcome & Author and year & $\begin{array}{c}\text { Duration of } \\
\text { exposure (h:min) }\end{array}$ & $\begin{array}{c}\text { Hypobaric } \\
\text { hypoxia result } \\
\text { [mean (SD)] }\end{array}$ & $\begin{array}{l}\text { Normobaric } \\
\text { hypoxia result } \\
\text { [mean (SD)] }\end{array}$ & $\begin{array}{l}\text { Difference } \\
\text { (HH - NH) }\end{array}$ & $P$ value & $\begin{array}{c}\text { Direction of } \\
\text { difference NH } \\
\text { compared to } \mathrm{HH}\end{array}$ \\
\hline \multicolumn{8}{|c|}{ Exhaled NO (PE NO) } \\
\hline & Hemmingsson 2009 & $\begin{array}{l}\text { 00:10 at each } \\
\text { ascending altitude }\end{array}$ & - & - & $\begin{array}{l}33 \% \text { mean } \\
\text { reduction } \\
\text { (at 5,000 m) }\end{array}$ & 0.002 & $\mathrm{NH}>\mathrm{HH}$ \\
\hline & Faiss 2013 & 01:00 & $9.5(5.0)$ & $14.9(9.2)$ & -5.4 & $<0.01$ & $\mathrm{NH}>\mathrm{HH}$ \\
\hline & Faiss 2013 & 08:00 & $8.8(5.3)$ & $14.1(7.4)$ & -5.3 & $<0.01$ & $\mathrm{NH}>\mathrm{HH}$ \\
\hline & Faiss 2013 & $16: 00$ & $7.9(4.5)$ & $14.7(8.6)$ & -6.8 & $<0.01$ & $\mathrm{NH}>\mathrm{HH}$ \\
\hline & Faiss 2013 & $24: 00: 00$ & $8.9(5.4)$ & $15.7(8.7)$ & -5.8 & $<0.01$ & $\mathrm{NH}>\mathrm{HH}$ \\
\hline \multicolumn{8}{|l|}{$\mathrm{RQ}$} \\
\hline & Self 2011 & 00:05 & $2.37(0.53)$ & $1.41(0.15)$ & 0.96 & 0.005 & $\mathrm{HH}>\mathrm{NH}$ \\
\hline \multicolumn{8}{|c|}{ Forced vascular conductance } \\
\hline & Miyagawa 2010 & 01:00-01:40 & - & - & - & $>0.05$ & NS \\
\hline \multicolumn{8}{|l|}{ Sweat rate } \\
\hline & Miyagawa 2010 & 01:00-01:40 & - & - & - & $>0.05$ & NS \\
\hline \multicolumn{8}{|c|}{$\begin{array}{l}\text { Oesophageal temperature } \\
\text { thresholds for increasing } \\
\text { forearm skin vascular } \\
\text { conductance }\end{array}$} \\
\hline & Miyagawa 2010 & 01:00-01:40 & - & - & - & $>0.05$ & NS \\
\hline \multicolumn{8}{|c|}{$\begin{array}{l}\text { Oesophageal temperature } \\
\text { thresholds for increasing } \\
\text { sweat rate }\end{array}$} \\
\hline & Miyagawa 2010 & 01:00-01:40 & - & - & - & $>0.05$ & NS \\
\hline \multicolumn{8}{|c|}{ Oesophageal temperature } \\
\hline & Miyagawa 2010 & 01:05 & 36.63 & 36.61 & 0.02 & $>0.05$ & NS \\
\hline & Miyagawa 2010 & 01:10 & 37.12 & 37.11 & 0.01 & $>0.05$ & NS \\
\hline & Miyagawa 2010 & 01:40 & 37.95 & 37.96 & -0.01 & $>0.05$ & NS \\
\hline \multicolumn{8}{|c|}{ Skin temperature } \\
\hline & Miyagawa 2010 & 01:05 & 33.37 & 33.47 & -0.1 & $>0.05$ & NS \\
\hline & Miyagawa 2010 & 01:10 & 33.35 & 33.43 & -0.08 & $>0.05$ & NS \\
\hline & Miyagawa 2010 & $01: 40$ & 34.44 & 34.59 & -0.15 & $>0.05$ & NS \\
\hline \multicolumn{8}{|c|}{ Urine vol (ml) } \\
\hline & Loeppky $2005^{a}$ & 10:00 & - & - & - & 0.005 & $\mathrm{HH}>\mathrm{NH}$ \\
\hline & Tucker $1983^{b}$ & $\begin{array}{l}\text { 02:20 including } \\
00: 15 \text { build }^{d}\end{array}$ & -1.6 & 0.1 & -1.7 & - & $\mathrm{NH}>\mathrm{HH}$ \\
\hline \multicolumn{8}{|c|}{ Plasma volume } \\
\hline & Miyagawa 2010 & 01:00-01:40 & - & - & - & $>0.05$ & NS \\
\hline & Loeppky 2005 & 10:00 & - & - & $-6 \%$ & $0.002^{* *}$ & $\mathrm{HH}>\mathrm{NH}$ \\
\hline \multicolumn{8}{|l|}{ GFR } \\
\hline & Loeppky 2005 & 10:00 & - & - & - & $>0.05$ & NS \\
\hline \multicolumn{8}{|l|}{ Plasma K ${ }^{+}$} \\
\hline & Loeppky 2005 & 10:00 & - & - & - & 0.003 & $\mathrm{NH}>\mathrm{HH}$ \\
\hline \multicolumn{8}{|l|}{ Plasma $\mathrm{Na}^{+}$} \\
\hline & Loeppky 2005 & 10:00 & - & - & - & 0.006 & $\mathrm{NH}>\mathrm{HH}$ \\
\hline \multicolumn{8}{|c|}{ Plasma renin activity (PRA) } \\
\hline & Loeppky $2005^{a}$ & $10: 00$ & - & - & - & $<0.05$ & $\mathrm{HH}>\mathrm{NH}$ \\
\hline
\end{tabular}


Table 6 Additional physiological variables (Continued)

\begin{tabular}{|c|c|c|c|c|c|c|c|}
\hline \multicolumn{8}{|l|}{$\overline{\text { Plasma aldosterone }}$} \\
\hline & Loeppky $2005^{\mathrm{a}}$ & $10: 00$ & - & - & - & $<0.001$ & $\mathrm{NH}>\mathrm{HH}$ \\
\hline \multicolumn{8}{|c|}{ Free water clearance $\left(\mathrm{CH}_{2} \mathrm{O}\right)$} \\
\hline & Loeppky $2005^{a}$ & $10: 00$ & - & - & - & $<0.05$ & $\mathrm{HH}>\mathrm{NH}$ \\
\hline \multicolumn{8}{|l|}{$\mathrm{ACTH}$} \\
\hline & Loeppky $2005^{a}$ & 10:00 & - & - & - & 0.18 & NS \\
\hline \multicolumn{8}{|l|}{ ADH } \\
\hline & Loeppky 2005 & 10:00 & - & - & - & $>0.05$ & NS \\
\hline \multicolumn{8}{|l|}{ ANP } \\
\hline & Loeppky 2005* & $10: 00$ & - & - & - & 0.97 & NS \\
\hline \multicolumn{8}{|l|}{ Blood base excess } \\
\hline & Loeppky 2005 & $10: 00$ & - & - & - & $>0.05$ & NS \\
\hline \multicolumn{8}{|l|}{ Urine $\mathrm{Na}^{+} / \mathrm{K}^{+}$} \\
\hline & Loeppky 2005 & $10: 00$ & - & - & - & 0.7 & NS \\
\hline \multicolumn{8}{|l|}{ Catecholamines } \\
\hline & Miyagawa 2010 & 01:00-01:40 & - & - & - & $>0.05$ & NS \\
\hline & Loeppky 2005 & 10:00 & - & - & - & 0.43 & NS \\
\hline \multicolumn{8}{|l|}{ Haematocrit } \\
\hline & Miyagawa 2010 & 01:00-01:40 & - & - & - & $>0.05$ & NS \\
\hline & Tucker $1983^{b}$ & $\begin{array}{l}\text { 02:20 including } \\
00: 15 \text { build }^{d}\end{array}$ & 0.7 & 0.7 & 0 & & NS \\
\hline \multicolumn{8}{|c|}{ Haemoglobin concentration } \\
\hline & Savourey 2007 & 00:10 build ${ }^{d}, 00: 05$ & - & - & - & $>0.05$ & NS \\
\hline & Savourey 2007 & $00: 10$ build $^{d}, 00: 30$ & - & - & - & $>0.05$ & NS \\
\hline & Miyagawa 2010 & 01:00-01:40 & - & - & - & $>0.05$ & NS \\
\hline \multicolumn{8}{|l|}{$\begin{array}{l}\text { Plasma osmolarity } \\
(\mathrm{mOsm})\end{array}$} \\
\hline & Miyagawa 2010 & 01:00-01:40 & - & - & - & $>0.05$ & NS \\
\hline & Tucker $1983^{b}$ & $\begin{array}{l}\text { 02:20 including } \\
00: 15 \text { build }^{d}\end{array}$ & -0.4 & -1 & 0.6 & - & $\mathrm{HH}>\mathrm{NH}$ \\
\hline \multicolumn{8}{|l|}{$\mathrm{pH}$} \\
\hline & Savourey 2003 & $00: 10$ build $^{d}, 00: 30$ & 7.46 (SEM 0.03) & 7.44 (SEM 0.02) & 0.02 & $\leq 0.05$ & Borderline $\mathrm{HH}>\mathrm{NH}$ \\
\hline & Savourey 2007 & $00: 10$ build $^{d}, 00: 30$ & $7.45(0.04)$ & $7.44(0.04)$ & 0.01 & 0.02 & $\mathrm{HH}>\mathrm{NH}$ \\
\hline \multicolumn{8}{|l|}{ CAL subjects } \\
\hline & Naughton 1995 & 00:52 & - & - & -0.02 & $>0.05$ & NS \\
\hline \multicolumn{8}{|l|}{ Control subjects } \\
\hline & Naughton 1995 & $00: 52$ & - & - & 0.01 & $>0.05$ & NS \\
\hline & Tucker $1983^{d}$ & $\begin{array}{l}\text { 02:20 including } \\
00: 15 \text { build }^{d}\end{array}$ & 0.047 & 0.015 & 0.032 & - & $\mathrm{HH}>\mathrm{NH}$ \\
\hline & Faiss 2013 & 24:00:00 & - & - & - & $<0.01$ & $\mathrm{NH}>\mathrm{HH}$ \\
\hline \multicolumn{8}{|c|}{ Urine osmolarity (mOsm) } \\
\hline & Tucker $1983^{c}$ & $\begin{array}{l}\text { 02:20 including } \\
00: 15 \text { build }^{b}\end{array}$ & 15 & 0.1 & 14.9 & - & $\mathrm{HH}>\mathrm{NH}$ \\
\hline \multicolumn{8}{|l|}{ Lactate $\mathrm{mmol} / \mathrm{kgH}_{2} \mathrm{O}$} \\
\hline & Miyagawa 2010 & 01:00-01:40 & - & - & - & $>0.05$ & NS \\
\hline
\end{tabular}


Table 6 Additional physiological variables (Continued)

\begin{tabular}{|c|c|c|c|c|c|c|c|}
\hline \multicolumn{8}{|l|}{$\overline{\text { Blood NO metabolites }}$} \\
\hline & Faiss 2013 & 01:00 & $31.6(19.6)$ & $27.7(7.3)$ & 3.9 & $<0.01$ & $\mathrm{HH}>\mathrm{NH}$ \\
\hline & Faiss 2013 & 08:00 & $28.1(18.9)$ & $32.7(9.7)$ & -4.6 & $<0.01$ & $\mathrm{NH}>\mathrm{HH}$ \\
\hline & Faiss 2013 & $16: 00$ & $24.2(16.3)$ & $30.2(7.1)$ & -6 & $<0.01$ & $\mathrm{NH}>\mathrm{HH}$ \\
\hline & Faiss 2013 & $24: 00$ & $22.85(16.2)$ & $28.9(6.9)$ & -6.05 & $<0.01$ & $\mathrm{NH}>\mathrm{HH}$ \\
\hline \multicolumn{8}{|l|}{ GPX (\% baseline) } \\
\hline & Faiss 2013 & 01:00 & $114(26)$ & $111(30)$ & 3 & $>0.05$ & NS \\
\hline & Faiss 2013 & 08:00 & $85(27)$ & $123(23)$ & -37 & $>0.05$ & NS \\
\hline & Faiss 2013 & $16: 00$ & $105(43)$ & $107(21)$ & -2 & $>0.05$ & NS \\
\hline & Faiss 2013 & 24:00 & $103(43)$ & $107(21)$ & -4 & $>0.05$ & NS \\
\hline \multicolumn{8}{|l|}{ MDA (\% baseline) } \\
\hline & Faiss 2013 & 01:00 & $117(40)$ & $92(36)$ & 25 & $>0.05$ & NS \\
\hline & Faiss 2013 & 08:00 & $103(62)$ & $111(35)$ & -8 & $>0.05$ & NS \\
\hline & Faiss 2013 & $16: 00$ & $111(56)$ & $116(55)$ & -5 & $>0.05$ & NS \\
\hline & Faiss 2013 & $24: 00$ & $108(52)$ & $97(51)$ & 11 & $>0.05$ & NS \\
\hline \multicolumn{8}{|c|}{ Nitrotyrosine (\% baseline) } \\
\hline & Faiss 2013 & 01:00 & $86(16)$ & $105(26)$ & -19 & $>0.05$ & NS \\
\hline & Faiss 2013 & 08:00 & 77 (35) & 75 (37) & 2 & $>0.05$ & NS \\
\hline & Faiss 2013 & $16: 00$ & $91(20)$ & $98(16)$ & -7 & $>0.05$ & NS \\
\hline & Faiss 2013 & $24: 00$ & $75(40)$ & $87(25)$ & -12 & $>0.05$ & NS \\
\hline \multicolumn{8}{|c|}{$\begin{array}{l}\text { Plasma advanced oxidation } \\
\text { protein products }\end{array}$} \\
\hline & Faiss 2013 & 01:00 & $120 \%$ & $13 \%$ & $107 \%$ & - & $\mathrm{NH}>\mathrm{HH}$ \\
\hline & Faiss 2013 & 24:00 & $260 \%$ & $88 \%$ & $172 \%$ & - & $\mathrm{NH}>\mathrm{HH}$ \\
\hline Superoxide dismutase & Faiss 2013 & $24: 00$ & - & - & $37 \%$ & - & $\mathrm{NH}>\mathrm{HH}$ \\
\hline $\begin{array}{l}\text { bDifference from controls } \\
\text { cSubjects were gradually } \\
\text { dWhen the subjects ente } \\
\text { specified amount of time } \\
\text { *P value calculated inclue } \\
{ }^{* *} P \text { value calculated inclu }\end{array}$ & $\begin{array}{l}\text { rreasing levels } \\
\text { mber, the envi } \\
\text { rget hypoxic d } \\
\text { ric normoxia. } \\
\text { aric normoxia }\end{array}$ & $\begin{array}{l}\text { over the } \\
\text { condition } \\
\text { ached. }\end{array}$ & $\begin{array}{l}\text { me until the ta } \\
\text { hat of normal s }\end{array}$ & $\begin{array}{l}\text { yypoxic dose } \\
\text { el but then }\end{array}$ & $\begin{array}{l}\text { eached. } \\
\text { radually }\end{array}$ & e more $h$ & c over the \\
\hline
\end{tabular}

the saturations were $4.08 \%$ lower in $\mathrm{HH}$ [18]. However, no differences were found in all five of the long studies $[12-14,16,22]$. The arterial blood saturations $\left(\mathrm{SaO}_{2}\right)$ were lower in $\mathrm{HH}$ in all three short studies $[6,17,18]$ but not in the two longer studies $[15,16]$. Arterial partial pressures of oxygen $\left(\mathrm{P}_{\mathrm{a}} \mathrm{O}_{2}\right)$ was lower in $\mathrm{NH}$ in one study [6], higher in $\mathrm{NH}$ in one study [17], and no different in two studies $[18,20]$ (Table 3: Oxygenation and ventilation). Only the two studies by Savourey et al. $[17,18]$ measured the end tidal fractions of $\mathrm{O}_{2}$ and these report discordant results. In 2003, Savourey et al. [17] found the end tidal fractions of $\mathrm{O}_{2}$ to be higher $(P<0.00001)$ in $\mathrm{HH}$ than $\mathrm{NH}$; however, in their 2007 [18] study following the same protocols, no difference was demonstrated $(P>0.05)$.

\section{Carbon dioxide $\left(\mathrm{CO}_{2}\right)$ clearance}

In three out of five studies, there was no difference in the end tidal partial pressure of $\mathrm{CO}_{2}\left(\mathrm{P}_{\mathrm{ET}} \mathrm{CO}_{2}\right)$ between $\mathrm{HH}$ and $\mathrm{NH}[17,18,22]$; however, one study [12] found it to be higher in $\mathrm{HH}$ and one found it to be lower in $\mathrm{HH}$ [13]. In four out of five studies, the $\mathrm{PaCO}_{2}$ levels were lower $[11,17,13]$ or the same [20] in $\mathrm{HH}$ compared to $\mathrm{NH}$. For example, one study found the difference in $\mathrm{PaCO}_{2}$ to be as large as $5.9 \mathrm{hPa}$ lower in $\mathrm{HH}$ [18]. Two studies measured the end tidal fractions of $\mathrm{CO}_{2}[17,18]$. In 2003, Savourey et al. [17] found the end tidal fractions of $\mathrm{CO}_{2}$ to be higher $(P<0.00001)$ in $\mathrm{HH}$ than $\mathrm{NH}$, whereas in the same group's 2007 study [18] (following the same protocols), no difference was demonstrated $(P>0.05)$. 


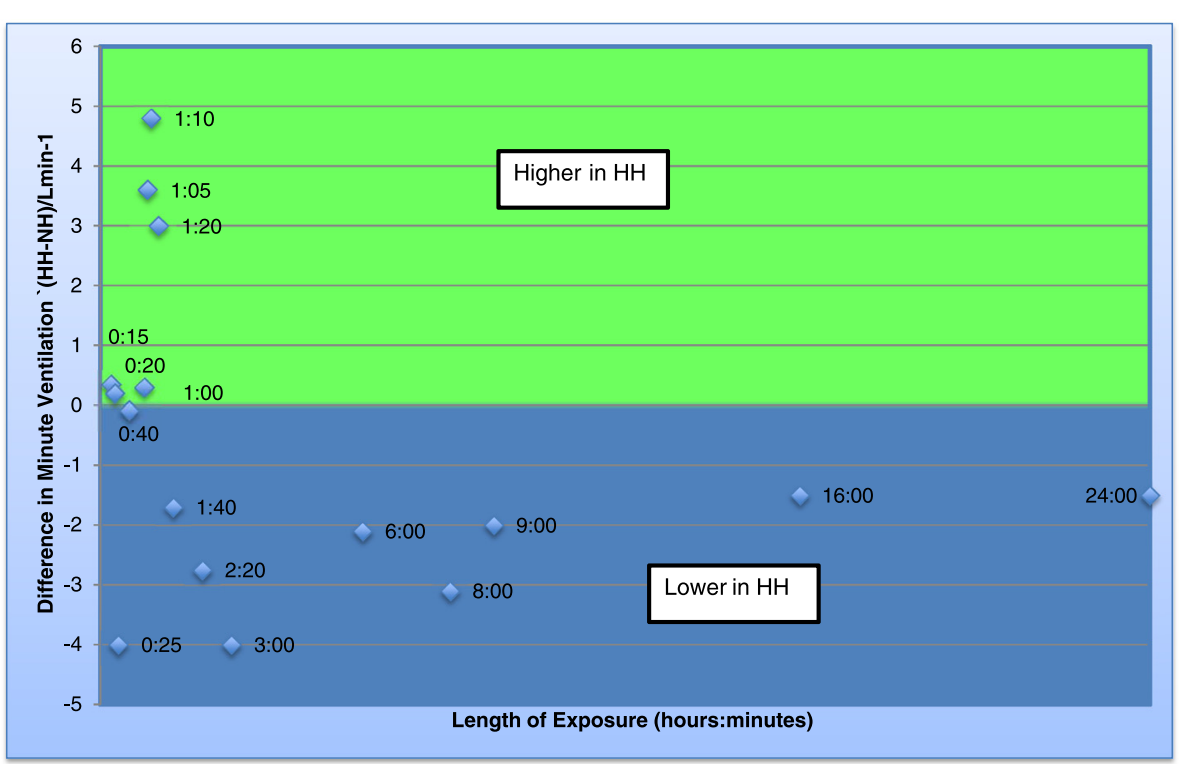

Figure 2 Graph of minute ventilation. Graph to show the difference in minute ventilation between the two environments over time. Each data point represents data obtained from a study and the number refers to the time point. If the data point is in the green area, the minute ventilation was found to be higher in $\mathrm{HH}$ but if in the blue area, the minute ventilation was found to be lower in $\mathrm{HH}$.

\section{Cardiovascular variables}

Seven studies were identified that reported physiological variables relating to the cardiovascular system. Three of these were long studies $[12,15,22]$ and four were short studies $[6,17,18,21]$ (Table 4: Cardiovascular variables). All seven studies measured heart rate (HR). Three found HR to be higher in $\mathrm{HH}[6,17,22]$, one found it to be lower [21], and the others found no differences $[12,15,18]$
(Figure 5: Graph of heart rates). Three studies measured blood pressure. Two found no difference in blood pressure between environments [12,15]; however, one other found it to be lower in $\mathrm{HH}$ than in NH [22]. Sympathetic drive, measured by a specific electrocardiogram (ECG) recorder of low- and high-frequency components of heart rate, was only investigated in one study [21] and was found to be higher in $\mathrm{HH}$ than in $\mathrm{NH}$.

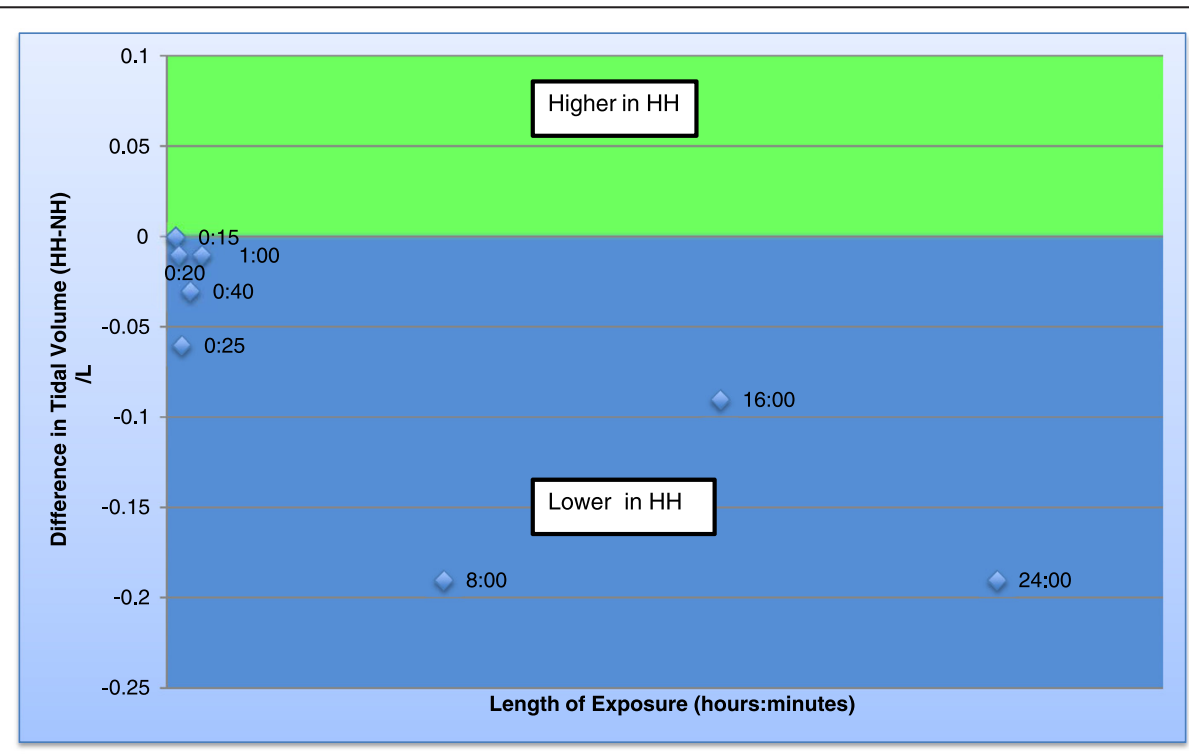

Figure 3 Graph of tidal volume. Graph to show the difference in tidal volume between the two environments over time. Each data point represents data obtained from a study and the number refers to the time point. If the data point is in the green area, the tidal volume was found to be higher in $\mathrm{HH}$ but if in the blue area, the tidal volume was found to be lower in $\mathrm{HH}$. 


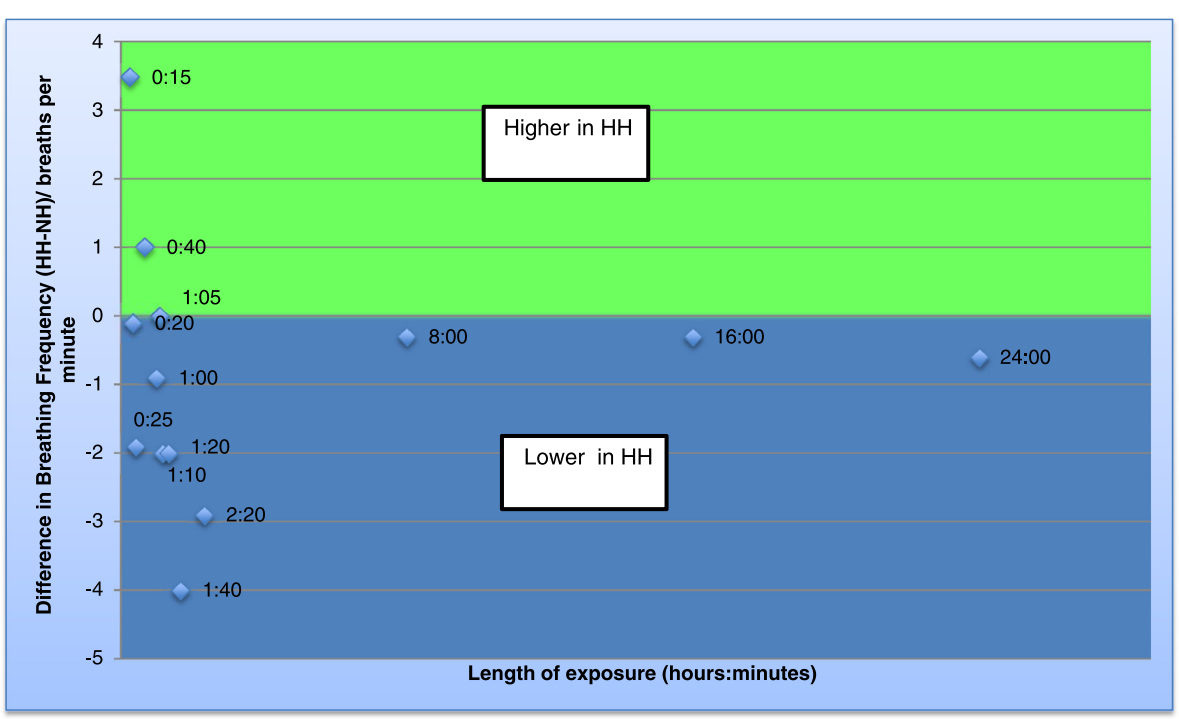

Figure 4 Graph of breathing frequency. Graph to show the difference in breathing frequency between the two environments over time. Each data point represents data obtained from a study and the number refers to the time point. If the data point is in the green area, the breathing frequency was found to be higher in $\mathrm{HH}$ but if in the blue area, the breathing frequency was found to be lower in $\mathrm{HH}$.

\section{Acute mountain sickness and neurology}

Four studies were found relating to acute mountain sickness (AMS) and neurological symptoms and signs, two of which were long studies $[14,16]$ and two of which were short studies [6,23] (Table 5: AMS and neurology). Two out of three studies [14,16] that measured AMS scores found that AMS was significantly worse in $\mathrm{HH}$ than in NH. Only one study [23] measured postural stability, which was significantly reduced in $\mathrm{HH}$ compared to $\mathrm{NH}$. Subjects deviated from the midline more in $\mathrm{HH}$ than $\mathrm{NH}$, and the speed with which movements occurred to correct their posture was slower.

\section{Additional physiological variables}

Six studies $[6,12,14,15,19,22]$ researched a variety of other physiological variables (Table 6: Additional physiological variables). In three out of five of these studies $[17,18,22]$, the plasma $\mathrm{pH}$ was higher in $\mathrm{HH}$ than $\mathrm{NH}$; however, one of

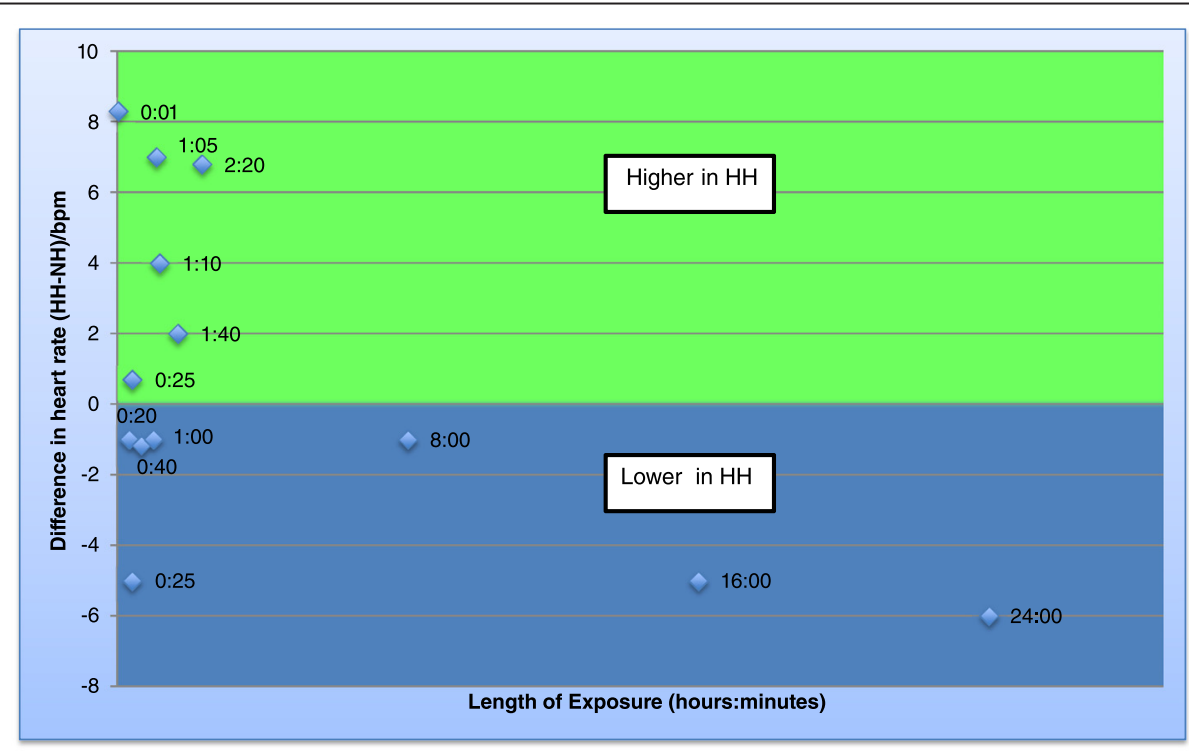

Figure 5 Graph of heart rates. Graph to show the difference in heart rate between the two environments over time. Each data point represents data obtained from a study and the number refers to the time point. If the data point is in the green area, the heart rate was found to be higher in $\mathrm{HH}$ but if in the blue area, the heart rate was found to be lower in $\mathrm{HH}$. 
the other papers found the $\mathrm{pH}$ to be higher in $\mathrm{NH}$ [12] (Figure 6: Graph of $\mathrm{pH}$ ). The greatest difference in $\mathrm{pH}$ found in a study was 0.032 [22]. Urine osmolarity was measured in only one study and was significantly higher in $\mathrm{HH}$ [22]. Additionally, the same study was the only one to measure the volume of urine produced and found it to be lower during $\mathrm{HH}$ exposure [22]. One study also measured $\mathrm{K}^{+}, \mathrm{Na}^{+}$and aldosterone concentrations in plasma and found they were higher in $\mathrm{NH}$ [14]. In recovery posthypoxic exposure, authors in [14] found that the urine volume, plasma renin activity and free water clearance were higher whilst aldosterone was lower after $\mathrm{HH}$ than $\mathrm{NH}$. This was the only study to measure these variables.

Only two studies $[12,19]$ measured exhaled NO levels and both found that they were lower in $\mathrm{HH}$ than in $\mathrm{NH}$. The greatest difference in exhaled NO found in these papers was $6.8 \mathrm{mmHg}$ [12]. Additionally, only one study measured systemic NO and the authors found it was lower in $\mathrm{HH}$ [12]. The same study was the only study to measure plasma-advanced oxidation protein products and superoxide dismutase. These were higher in $\mathrm{HH}$ [12]. Only one study [6] measured the respiratory quotient (RQ) and found it was higher in $\mathrm{HH}$ than $\mathrm{NH}$.

\section{Review: discussion}

\section{Summary of physiological findings}

We believe this is the first systematic review comparing the effect of $\mathrm{NH}$ and $\mathrm{HH}$ on human physiology.
Significant differences were demonstrated in variables relating to ventilation, NO, fluid retention, and in factors relating to AMS. For other variables, there was no consistent pattern across the reviewed studies.

\section{Oxygenation and ventilation parameters}

The majority of studies included data relating to oxygenation and ventilation. Oxygen saturations measured from pulse oximetry $\left(\mathrm{SpO}_{2}\right)$ and arterial blood gases correlated with each other. During short-term exposure, oxygen saturations were lower in $\mathrm{HH}[6,17]$. This was not the case for long-term exposure, perhaps as the initial process of acclimatisation to hypoxia had occurred $[18,20] . \mathrm{P}_{\mathrm{a}} \mathrm{O}_{2}$ did not differ at any stage.

A decreased minute ventilation and alveolar ventilation was seen in $\mathrm{HH}$. This finding is in accord with the smaller tidal volumes demonstrated in $\mathrm{HH}$; however, breathing frequencies varied $[12,13,17,18,21,22]$. Despite the lower $\mathrm{SpO}_{2}$ in $\mathrm{HH}$ initially, oxygen saturations were maintained at the same level in both environments over longer time periods. Interestingly, despite the reduction in ventilation, $\mathrm{P}_{\mathrm{ET}} \mathrm{CO}_{2}$ levels did not change significantly $[12,13,17,18,22]$.

Notably, Savourey et al. found different results in end tidal fractions of both $\mathrm{O}_{2}$ and $\mathrm{CO}_{2}$ between their two studies in 2003 [17] and 2007 [18]. This not only makes interpretation of their results very challenging but also highlights issues concerning reliability of studies (as discussed in Study quality section).

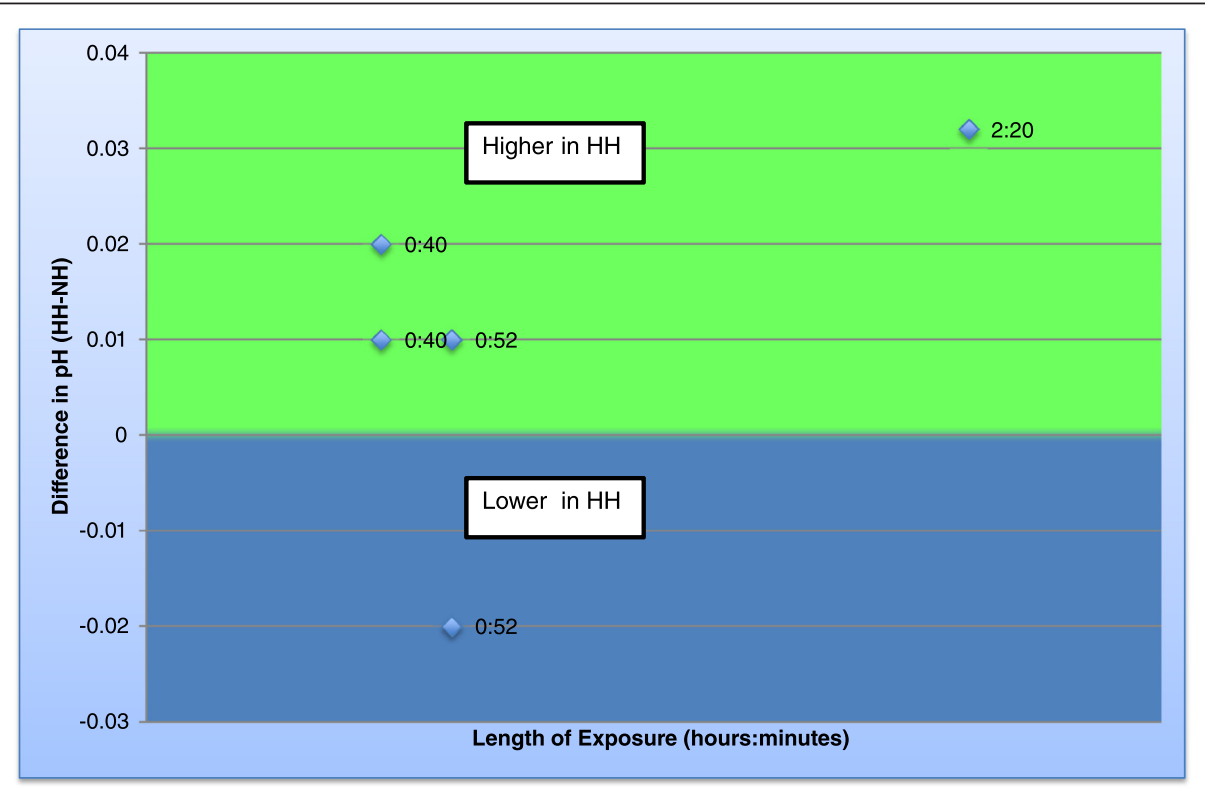

Figure 6 Graph of pHs. Graph to show the difference in pH between the two environments over time. Each data point represents data obtained from a study and the number refers to the time point. If the data point is in the green area, the $\mathrm{pH}$ was found to be higher in $\mathrm{HH}$ but if in the blue area, the $\mathrm{pH}$ was found to be lower in $\mathrm{HH}$. 


\section{Cardiovascular variables}

The majority of these variables were similar for $\mathrm{HH}$ and $\mathrm{NH}$. Heart rate and blood pressure results were inconsistent, although some data suggests that heart rate may be raised initially in $\mathrm{HH}[6,12,15,17,18,21,22]$.

\section{AMS and neurology variables}

Mountain sickness is an area of research that has attracted much interest and consequently the multiple variables that combine to make the Lake Louise AMS scoring system are commonly collected in these studies. In two of four studies, AMS severity was found to increase in $\mathrm{HH}$ compared with $\mathrm{NH}[14,16]$. Furthermore, in the one study that found no differences between environments [6], participants only had a 5-min exposure time, as opposed to 9- and 10-h exposures in other studies. Additionally, postural stability was significantly worse in $\mathrm{HH}$ [23]. The authors suggest that visual contrast sensitivity is lower in $\mathrm{HH}$ than in $\mathrm{NH}$ and that this may contribute to the postural stability [23].

\section{Other homeostatic variables}

Plasma $\mathrm{pH}$ appears to be higher in $\mathrm{HH}$ than $\mathrm{NH}$ $[17,18,22]$. The study [20] that did not find significant differences in $\mathrm{pH}$ between the two conditions was conducted at 1,829 and 2,438 $\mathrm{m}$ whereas the elevated $\mathrm{pH}$ values were from $4,500 \mathrm{~m}$, suggesting that the differences between the two conditions may be more pronounced at higher altitudes. Elevated $\mathrm{pH}$ in $\mathrm{HH}$ is surprising in the context of the finding that ventilation is reduced under these conditions.

Exhaled and systemic NO levels were lower in $\mathrm{HH}$ $[12,19]$. Faiss et al. [12] found increased oxidative stress in $\mathrm{HH}$ and attributed the systemic differences in $\mathrm{NO}$ bioavailability to this.

Many other physiological variables were measured; however, most of these were reported in only a single study. Thus, it is difficult to make conclusions without verification from other studies, and we have not considered these further.

\section{Study quality}

The search results reveal several issues relating to study design. Very few studies state the reliability of their measurements or performed a sample size calculation. It is therefore difficult to evaluate if they are adequately powered to identify a real difference between conditions, should such a difference exist. Given that differences in physiological responses between $\mathrm{NH}$ and $\mathrm{HH}$ conditions are likely quite small, large sample sizes would likely be required to avoid type II (false negative) errors. Additionally, by performing statistical analysis on a large number of variables over many time points, the risk of type I (false positive) errors increases.
Failure to account for the $\mathrm{P}_{\mathrm{H} 2 \mathrm{O}}$ leads to an overestimate of the hypoxic dose in $\mathrm{NH}$, such that incorrect partial pressure of inspired oxygen $\left(\mathrm{PiO}_{2}\right)$ may be attained [11]. In one study [6], a $\mathrm{NH}$ exposure equivalent to 7,620 $\mathrm{m}$ was described; however, the conditions were in fact closer to $7,010 \mathrm{~m}$ once $\mathrm{pH}_{2} \mathrm{O}$ was accounted for [11]. We have emphasised these differences by calculating, where possible, the $\mathrm{PiO}_{2}$ in the different hypoxic conditions (Table 2: Study design). We found the differences in $\mathrm{PiO}_{2}$ to be as much as $4 \mathrm{mmHg}$. The level of $\mathrm{CO}_{2}$ in the test environment was a potential source of error. Basualto-Alarcon et al. [21] highlight this issue in stating that different gas inflow rates into each hypoxic system allow different levels of $\mathrm{CO}_{2}$ accumulation. Additionally, they state that their $\mathrm{NH}$ environment may have been more hypercapnic because it had half the total volume of the hypobaric chamber. These control group contrast issues will either enhance or diminish the effect size and therefore the difference between $\mathrm{HH}$ and $\mathrm{NH}$. This may be of particular relevance to ventilator variables.

\section{Mechanisms for results}

Many hypothetical mechanisms have been proposed for the effect of low barometric pressure on physiology. These include intravascular bubble formation, increased alveolar deadspace, altered fluid permeability, changes in chemosensitivity, and a mismatch in ventilation and perfusion $[13,16,17]$. Although pressure may be the principle confounder between the two scenarios, we must also reflect that other factors may differ between $\mathrm{HH}$ and $\mathrm{NH}$, thus impacting participant's physiology. For example, the laboratory-based components of the studies reviewed were performed between $22^{\circ} \mathrm{C}$ and $25^{\circ} \mathrm{C}$, a temperature likely to be far warmer that experiences at $4,000 \mathrm{~m}$ in a field laboratory. Such differences in ambient temperature may alter physiological mechanisms such as the degree of peripheral vasoconstriction, NO metabolism or the production of reactive oxidative species [24].

As highlighted, the duration of the hypoxic exposure impacts on the results obtained. Different physiological systems will have different response rates for adaptation to hypoxia [25]. For some physiological parameters, the short study durations may not be long enough for differences between $\mathrm{NH}$ and $\mathrm{HH}$ to be elicited. Studies reporting repeated measures over time provide a window on this phenomenon. For example, in the 1997 study by Loeppky et al. [13], where no differences in minute ventilation were reported after 30 or $60 \mathrm{~min}$ of hypoxic exposure, significant differences were evident after $3 \mathrm{~h}$ of exposure. Additionally, Savourey et al. $[17,18]$ initially found lower $\mathrm{P}_{\mathrm{ET}} \mathrm{O}_{2}$ and $\mathrm{P}_{\mathrm{ET}} \mathrm{CO}_{2}$ in $\mathrm{HH}$ than $\mathrm{NH}$ but then no difference in prolonged exposure. This may be because during $\mathrm{HH}$ exposure, the ambient 
partial pressure of nitrogen $\left(\mathrm{P}_{\mathrm{N} 2}\right)$ is initially lower than the body's and therefore nitrogen $\left(\mathrm{N}_{2}\right)$ initially diffuses from the tissues to the alveoli [5]. Until this equilibrium is achieved, the arterial oxygen content, $\mathrm{P}_{\mathrm{A}} \mathrm{O}_{2}$, and the arterial carbon dioxide content, $\mathrm{P}_{\mathrm{A}} \mathrm{CO}_{2}$, are lowered as a result of the relatively higher $\mathrm{P}_{\mathrm{A}} \mathrm{N}_{2}$ in $\mathrm{HH}$ than $\mathrm{NH}$.

Loeppky et al. [13] also suggests that an initial increase in $\mathrm{CO}_{2}$ produced in $\mathrm{HH}$ compared to $\mathrm{NH}$ might be due to microbubble formation similar to the nitrogen bends in divers. This emphasises the importance of study duration on physiological response and the problems inherent in comparing studies of different hypoxia exposure times.

If there are indeed differences between $\mathrm{HH}$ and $\mathrm{NH}$, at what equivalent altitude do they become apparent? Most of the studies have been carried out at 4,500 $\mathrm{m}$ (or equivalent), but Naughton et al.'s study [20] performed at 1,829 and $2,438 \mathrm{~m}$ was unable to find any significant differences between $\mathrm{HH}$ and $\mathrm{NH}$. These altitudes correspond to $\mathrm{PO}_{2}$ values of $118 \mathrm{mmHg}(15.7 \mathrm{kPa})$ and $108 \mathrm{mmHg}(14.4 \mathrm{kPa})$ [25] respectively, and it is possible that these altitudes were not high enough to elicit differences in the measured variables. Significant differences between the effects of $\mathrm{NH}$ and $\mathrm{HH}$ may impact the interpretation and application of results from studies at high altitude where the change in pressure may be a confounding influence in the evaluation of physiological responses to high altitude.

\section{Strengths and limitations of this study}

Although this is the first systematic review to summarise crossover studies comparing physiological responses to hypobaric and normobaric hypoxia, other publications have come to similar conclusions on the topic. Millet et al. [10] stress the importance of disentangling hypoxia and hypobaria and Fulco et al. [26] highlight the need for further investigations into $\mathrm{NH}$ versus $\mathrm{HH}$, for particular application to pre-acclimatisation strategies.

The strengths of this systematic review include the clear research question, comprehensive search strategy and consistent methods used for identifying eligible manuscripts and extracting data. Limitations of this review include the focus on crossover studies but are predominantly related to the quantity and quality of the underlying literature. There are few studies that compare $\mathrm{HH}$ and $\mathrm{NH}$ and the number of participants in each study is small. Whilst several of these studies report interesting differences between $\mathrm{HH}$ and $\mathrm{NH}$, there is marked inconsistency in the reported results. This may be due to a number of other factors including heterogeneity of study design, duration and magnitude of hypoxic dose and outcome reporting. Furthermore, the reporting of multiple phenotypes in each study without correction for repeat testing may be associated with an increased likelihood of type 1 errors. Conversely, the small sample sizes may be associated with an increased likelihood of type 2 errors.

As mentioned, the studies were heterogeneous by design. For example, they differed in regard to the subjects' prior exposure to altitude. In two of the studies reviewed $[16,22]$, the subjects lived between 1,500 and $1,600 \mathrm{~m}$ and so may have been partially acclimatised to high altitude. It is not clear whether the same effects would be seen in partially and not acclimatised subjects.

Finally, the self-reported nature of AMS scores could be associated with inconsistent responses from participants. In the study by Self et al. [6], there was a disparity between post-hypoxia interview responses and the responses during hypoxic exposure. There is no gold standard method for these types of studies and so there is much variability due to the methodology employed.

\section{Conclusions}

We present an overview of the current available literature regarding crossover studies relating to the different effects of $\mathrm{HH}$ and $\mathrm{NH}$ on human physiology. This systematic review is the first to compare the effects of a $\mathrm{NH}$ and $\mathrm{HH}$ environment on human physiology. Several studies reported a number of variables (e.g. minute ventilation and NO levels) that were different between the two conditions, lending support to the notion that true physiological difference are indeed present. However, the presence of confounding factors such as time spent in hypoxia, temperature, and humidity, and the limited statistical power due to small sample sizes, limit the conclusions that can be drawn from these findings.

Standardisation of study methods and reporting may aid interpretation of future studies and thereby improve the quality of data in this area. This is important to improve the quality of data that is used both for improving understanding of hypoxia tolerance, both at altitude and in the clinical setting.

\section{Abbreviations}

ACTH: Adrenocorticotropic hormone; ADH: Anti-diuretic hormone; AMS: Acute mountain sickness; ANP: Anti-naturetic protein; Bf: Respiratory rate; $\mathrm{BP}$ : Blood pressure; $\mathrm{CO}_{2}$ : Carbon dioxide; $\mathrm{CO}$ : Cardiac output; ECG: Electrocardiogram; FetCO $\mathrm{F}_{2}$ : End tidal fraction of carbon dioxide; $\mathrm{FetO}_{2}$ : End tidal fraction of oxygen; exNO: Exhaled nitric oxide levels; GFR: Glomerular filtration rate; GPX: Glutathione peroxidase; Hb conc: Haemoglobin concentration; Hct: Haematocrit; HR: Heart rate; LF/HF: Heart rate variability; $\mathrm{pH}_{2} \mathrm{O}$ : Humidity; $\mathrm{HH}$ : Hypobaric hypoxia; HCR: Hypoxic cardiac response; HVR: Hypoxic ventilatory response; NO: Nitric oxide; $\mathrm{N}_{2}$ : Nitrogen; $\mathrm{NH}$ : Normobaric hypoxic; $\mathrm{SpO}_{2}$ : Pulse oximetry; $\mathrm{P}_{\mathrm{N} 2}$ : Partial pressure of nitrogen; $\mathrm{PO}_{2}$ : Partial pressure of oxygen; $\mathrm{PaCO}_{2}$ : Arterial partial pressures of carbon dioxide; $\mathrm{PaO}_{2}$ : Arterial partial pressures of oxygen; $\mathrm{PetO}_{2}$ : End tidal partial pressure of oxygen; $\mathrm{P}_{\mathrm{ET}} \mathrm{CO}_{2}$ : End tidal partial pressure of $\mathrm{CO}_{2}$; Plasma $\mathrm{K}^{+}$: Plasma potassium concentration; plasma $\mathrm{Na}^{+}$: Plasma sodium concentration; $\mathrm{RQ}$ : Respiratory quotient; $\mathrm{SaO}_{2}$ : Arterial blood saturations; SV: Stroke volume; urine $\mathrm{Na}^{+} / \mathrm{K}^{+}$: Urine sodium-potassium ratio; $V A$ : Alveolar ventilation; $\mathrm{VCO}_{2}$ : Volume of $\mathrm{CO}_{2}$ produced; $\mathrm{VE}$ : Ventilation; $\mathrm{VO}_{2}$ : Volume of oxygen consumed; $\mathrm{VT}$ : Tidal volume. 


\section{Competing interests}

The authors declare that they have no competing interests.

\section{Authors' contributions}

EGK, PH and MG conceived the idea of the review. JC, EGK and PH planned the methodology and JC carried out the systematic review of the literature. The results were then checked by EGK and all authors discussed them. The review was drafted by $\mathrm{JC}$ and then commented on by all authors. All authors read and approved the final manuscript.

\section{Acknowledgements}

We would like to thank Caroline De Brun.

\section{Author details}

${ }^{1}$ University College London Centre for Altitude Space and Extreme Environment Medicine, UCLH NIHR Biomedical Research Centre, Institute of Sport and Exercise Health, 170 Tottenham Court Road, London W1T 7HA, UK. ${ }^{2}$ Integrative Physiology and Critical IIIness Group, Clinical and Experimental Sciences, Mailpoint 810, Sir Henry Wellcome Laboratories, Faculty of Medicine, University of Southampton, University Hospital Southampton NHS Foundation Trust, Tremona Road, Southampton SO16 6YD, UK. ${ }^{3}$ Anaesthesia and Critical Care Research Unit, University Hospital Southampton NHS Foundation Trust, Mailpoint 27, D Level, Centre Block, Tremona Road, Southampton SO16 6YD, UK. ${ }^{4}$ NIHR Southampton Respiratory Biomedical Research Unit, Southampton SO16 5ST, UK.

Received: 2 September 2014 Accepted: 18 December 2014 Published online: 26 February 2015

\section{References}

1. Grocott M, Montgomery H, Vercueil A. High-altitude physiology and pathophysiology: implications and relevance for intensive care medicine. Crit Care. 2007;11:203.

2. Grant S, MacLeod N, Kay JW, Watt M, Patel S, Paterson A, et al. Sea level and acute responses to hypoxia: do they predict physiological responses and acute mountain sickness at altitude? Brit J Sport Med. 2002;36:141-6.

3. Millet GP, Faiss R, Brocherie F, Girard O. Hypoxic training and team sports: a challenge to traditional methods? Brit J Sport Med. 2013;47:6-7.

4. DeHart RLE. Fundamentals of aerospace medicine. 2nd ed. Baltimore: Williams and Wilkins; 1996. p. 90-1.

5. Conkin J, Wessel JH. Critique of the equivalent air altitude model. Aviat Space Envir Md. 2008;79:975-82.

6. Self DA, Mandella JG, Prinzo OV, Forster EM, Shaffstall RM. Physiological equivalence of normobaric and hypobaric exposures of humans to 25,000 feet (7620 m). Aviat Space Envir Md. 2011;82:97-103.

7. Debevec T, Millet GP. Discerning normobaric and hypobaric hypoxia: significance of exposure duration. J Appl Physiol. 2014;116:1255-5.

8. Olivier G, Koehle MS, Guenette JA, Verges S, Chapman RF, Conkin J, et al. Comments on point: counterpoint: hypobaric hypoxia induces/does not induce different responses from normobaric hypoxia. J Appl Physiol. 2012;112:1788-94.

9. Muza SR. Military applications of hypoxic training for high-altitude operations. Med Sci Sports Exerc. 2007;39:1625-31.

10. Millet GP, Faiss R, Pialoux V. Evidence for differences between hypobaric and normobaric hypoxia is conclusive. Exerc Sport Sci Rev. 2013;41:133.

11. Conkin J. PH2O and simulated hypobaric hypoxia. Aviat Space Envir Md. 2011;82:1157-8.

12. Faiss R, Pialoux V, Sartori C, Faes C, Deriaz O, Millet GP. Ventilation, oxidative stress, and nitric oxide in hypobaric versus normobaric hypoxia. Med Sci Sports Exerc. 2013;45:253-60.

13. Loeppky JA, Icenogle M, Scotto P, Robergs R, HinghoferSzalkay H, Roach RC. Ventilation during simulated altitude, normobaric hypoxia and normoxic hypobaria. Resp Physiol. 1997;107:231-9.

14. Loeppky JA, Roach RC, Maes D, Hinghofer-Szalkay H, Roessler A, Gates L, et al. Role of hypobaria in fluid balance response to hypoxia. High Alt Med Biol. 2005;6:60-71.

15. Miyagawa K, Kamijo Y, Ikegawa S, Goto M, Nose H. Reduced hyperthermiainduced cutaneous vasodilation and enhanced exercise-induced plasma water loss at simulated high altitude $(3,200 \mathrm{~m})$ in humans. J Appl Physiol. 2011;110:157-65.
16. Roach RC, Loeppky JA, Icenogle MV. Acute mountain sickness: increased severity during simulated altitude compared with normobaric hypoxia. J Appl Physiol. 1996;81:1908-10.

17. Savourey G, Launay JC, Besnard Y, Guinet A, Travers S. Normo- and hypobaric hypoxia: are there any physiological differences? Eur J Appl Physiol. 2003;89:122-6.

18. Savourey G, Launay JC, Besnard Y, Guinet-Lebreton A, Alonso A, Sauvet F, et al. Normo or hypobaric hypoxic tests: propositions for the determination of the individual susceptibility to altitude illnesses. Eur J Appl Physiol. 2007;100:193-205.

19. Hemmingsson T, Linnarsson D. Lower exhaled nitric oxide in hypobaric than in normobaric acute hypoxia. Resp Physiol Neurobi. 2009;169:74-7.

20. Naughton MT, Rochford PD, Pretto JJ, Pierce RJ, Cain NF, Irving LB. Is normobaric simulation of hypobaric hypoxia accurate in chronic air-flow limitation. Am J Resp Crit Care. 1995;152:1956-60.

21. Basualto-Alarcon C, Rodas G, et al. Cardiorespiratory parameters during submaximal exercise under acute exposure to normobaric and hypobaric hypoxia. Apunts Med Esport. 2012;47:65-72.

22. Tucker A, Reeves JT, Robertshaw D, Grover RF. Cardiopulmonary response to acute altitude exposure —water loading and denitrogenation. Resp Physiol. 1983:54:363-80.

23. Degache F, Larghi G, Faiss R, Deriaz O, Millet G. Hypobaric versus normobaric hypoxia: same effects on postural stability? High Alt Med Biol. 2012;13:40-5.

24. Isa KBM, Kawasaki N, Ueyama K, Sumii T, Kudo S. Effect of cold exposure and shear stree on endothelial nitric oxide synthase activation. Biochem Biophys Res Commun. 2011;412:318-22.

25. West JB, Milledge JS, Schoene RB, Luks A. High altitude medicine and physiology. 5th ed. Boca Raton: CRC Press/Taylor \& Francis Group; 2013.

26. Fulco CS, Beidleman BA, Muza SR. Effectiveness of preacclimatization strategies for high-altitude exposure. Exerc Sport Sci Rev. 2013;41:55-63.

\section{Submit your next manuscript to BioMed Central and take full advantage of:}

- Convenient online submission

- Thorough peer review

- No space constraints or color figure charges

- Immediate publication on acceptance

- Inclusion in PubMed, CAS, Scopus and Google Scholar

- Research which is freely available for redistribution 